\title{
INTERACTING BROWNIAN PARTICLES AND GIBBS FIELDS ON PATHSPACES
}

\author{
DAVID DEREUDRE ${ }^{1}$
}

\begin{abstract}
In this paper, we prove that the laws of interacting Brownian particles are characterized as Gibbs fields on pathspace associated to an explicit class of Hamiltonian functionals. More generally, we show that a large class of Gibbs fields on pathspace corresponds to Brownian diffusions. Some applications to time reversal in the stationary and non stationary case are presented.
\end{abstract}

Mathematics Subject Classification. 60J60, 60K35, 60G55, 60G60, 82B21, 82C22.

Received July 30, 2002. Revised January 29, 2003.

\section{INTRODUCTION AND FRAMEWORK}

\subsection{Introduction}

The Gibbsian nature of infinite-dimensional diffusions on the infinite product of pathspaces has been first considered by Deuschel in 1987 [7]. Since this time, many papers developped this point of view (see for example $[3,4,25])$. Cattiaux et al. proved in [3], using an integration by parts formula on the pathspace, that the set of infinite-dimensional Brownian diffusions indexed by the lattice $\mathbb{Z}^{d}$ and the set of Gibbs measures on $C([0,1] ; \mathbb{R})^{\mathbb{Z}^{d}}$ are in one-to-one correspondance.

The generalization of these results to continuous models is our principal aim. In this paper, indistinguishable particles diffuse in $\mathbb{R}^{d}$ and interact in a way which depends only on their relative positions. So, this infinitedimensional diffusion can be seen as a point process on $\mathcal{C}:=C\left([0,1] ; \mathbb{R}^{d}\right)$. Our principal result, in dimension $d \leq 3$, is the equivalence between the following two properties: to be a infinite-dimensional gradient diffusion for a continuous model and to be a Gibbs field on $\mathcal{C}$.

Now, we describe the structure of the paper.

In the first part, we show that an interacting Brownian particle system is a solution of the following stochastic differential system:

$$
\left\{\begin{array}{l}
\mathrm{d} X_{i}(t)=\mathrm{d} W_{i}(t)-\frac{1}{2} \sum_{j \neq i} \nabla \varphi\left(X_{i}(t)-X_{j}(t)\right) \mathrm{d} t, \quad i \in \mathbb{N}^{*}, t \in[0,1] \\
X_{i}(0)=x_{i}, \quad i \in \mathbb{N}^{*}
\end{array}\right.
$$

\footnotetext{
Keywords and phrases. Point measure on pathspace, Gibbs field, interacting Brownian particles, integration by parts formula, Campbell measure.

1 Centre de Mathématiques Appliquées, UMR 7641, École Polytechnique, 91128 Palaiseau Cedex, France;

e-mail: dereudre@cmapx.polytechnique.fr
} 
where $\left(W_{i}\right)_{i \in \mathbb{N}^{*}}$ are independent Brownian motions with values in $\mathbb{R}^{d},\left(x_{i}\right)_{i \in \mathbb{N}^{*}}$ is a locally finite sequence of points in $\mathbb{R}^{d}$ and $\varphi$ is a regular symmetric potential with compact support. We represent a solution to (1) by the following point process on $\mathcal{C}: \Gamma=\sum_{i \in \mathbb{N}^{*}} \delta_{X_{i}}$.

The system (1) has first been studied by Lang in his fundamental paper [21]. He proved the existence of a unique strong solution in the stationary case. Since we also consider the non-stationary framework, we will use here Fritz's results, recalled in Theorem 1.1: he proved existence of a unique strong solution for the system (1) for every deterministic initial condition $\gamma=\sum_{i} \delta_{x_{i}}$ with finite logarithmic fluctuation energy $\mathcal{E}(\gamma)$ (see Def. 14).

In the second part, after establishing uniform bounds on weighted particle fluctuations, we prove in Theorems 2.1 and 2.2 the equivalence between the following assertions:

- to be the law on $\mathcal{M}(\mathcal{C})$ of the solution of system (1), with initial law given by a Gibbs field on $\mathbb{R}^{d}$ associated to the Hamiltonian $h$;

- to be a Gibbs field on $\mathcal{C}$ associated to the Hamiltonian $h(X(0), \Gamma(0))+H^{\Phi}(X, \Gamma)$, sum of a term induced by the initial law and a purely dynamic ones, the explicit form of $H^{\Phi}$ being given in (26).

Definition of Gibbs fields on $\mathbb{R}^{d}$ and $\mathcal{C}$, and Hamiltonians are given in Section 1.2.

In the third part, we prove in Theorem 3.1 that every Gibbs field $P$ on $\mathcal{C}$ associated to a regular local Hamiltonian $H$ is the law of an infinite-dimensional diffusion $-c f$. $(44)-$ with a drift $\left(\beta_{t}(X, \Gamma)\right)_{t \in[0,1]}$ of the following explicit form:

$$
\beta_{t}=-C_{P}^{!}\left(D_{t} H \mid \mathcal{F}_{t}\right),
$$

where $D$ is the Malliavin derivation operator and $C_{P}^{!}$the reduced Campbell measure associated to $P$ ( $c f$. Def. 8). The principal tool used to prove this result is the integration by parts formula (37) under the reduced Campbell measure.

Then, we present two applications of the above results. The first one is a proof that the tempered reversible probability measures of system (1) are the canonical Gibbs fields on $\mathbb{R}^{d}$ associated to the Hamiltonian

$$
h^{\varphi}(x, \gamma)=\sum_{y \in \gamma} \varphi(x-y)
$$

This was already proved in [22], but under much stronger assumptions than here. The second application is an analysis of time reversal for a general Gibbs field $P$ on $\mathcal{C}$. We prove that the projection of $P$ at time $t \geq 0$ is still a Gibbs fields on $\mathbb{R}^{d}$ for local Hamiltonian $h_{t}$, and we present in Theorem 3.2 a relation between $h_{t}$ and the forward and backward drifts associated to $P$. This formula is a generalization of results obtained by Föllmer and Wakolbinger [10] (cf. [24] too).

\subsection{Definitions and notations}

\subsubsection{State spaces and their probability measures}

If $\mathbb{X}$ denotes a polish space endowed with the Borel $\sigma$-algebra $\sigma(\mathbb{X})$, then $\mathcal{B}(\mathbb{X})$ and $\mathcal{S}(\mathbb{X})$ will denote subsets of $\sigma(\mathbb{X}) . \mathcal{M}(\mathbb{X})$ is the subset of the integer-valued measures $\Gamma$ on $\mathbb{X}$ such that, for every $\Lambda \in \mathcal{B}(\mathbb{X}), \Gamma(\Lambda) \in \mathbb{N}^{*}$ and for every $S \in \mathcal{S}(\mathbb{X}), \Gamma(S) \leq 1 ; \mathcal{M}(\mathbb{X})$ is endowed with the $\sigma$-algebra $\sigma(\mathcal{M}(\mathbb{X}))$ generated by the sets $\{\Gamma \in \mathcal{M}(\mathbb{X}), \Gamma(\Lambda)=n\}, n \in \mathbb{N}^{*}, \Lambda \in \mathcal{B}(\mathbb{X})$. A measure $\mu$ is called $\sigma$-finite if it is finite on the elements of $\mathcal{B}(\mathbb{X})$, and we denote by $\mathcal{P}(\mathcal{M}(\mathbb{X}))$ the set of probability measures on $\mathcal{M}(\mathbb{X})$.

For all $\Gamma \in \mathcal{M}(\mathbb{X}), P \in \mathcal{P}(\mathcal{M}(\mathbb{X}))$ and $\Lambda \in \sigma(\mathbb{X})$, we denote by $\Gamma_{\Lambda}$ the projection of $\Gamma$ on $\Lambda$ and $P_{\Lambda}$ the projection of $P$ on $\mathcal{M}(\Lambda)$. $\Pi^{\mu}$ denotes the Poisson process on $\mathbb{X}$ with intensity $\mu$, a $\sigma$-finite measure on $\mathbb{X}$. In the following, $\mathbb{X}$ will be either $\mathbb{R}^{d}$ or $\mathcal{C}$, the set of continuous paths from $[0,1]$ to $\mathbb{R}^{d}$ endowed with the uniform norm.

In the case $\mathbb{X}=\mathbb{R}^{d}$, we take $\mathcal{B}\left(\mathbb{R}^{d}\right)$ (respectively $\mathcal{S}\left(\mathbb{R}^{d}\right)$ ) equal to the set of usual bounded of subsets of $\mathbb{R}^{d}$ (respectively the set of single points of $\mathbb{R}^{d}$ ). Thus, $\mathcal{M}\left(\mathbb{R}^{d}\right)$ is the set of simple integer-valued measures on $\mathbb{R}^{d}$. Moreover, on $\mathbb{R}^{d}$, we substitute the notations $X, \Gamma, \Pi$ by $x, \gamma, \pi$ and we denote by $\lambda$ the Lebesgue measure on $\mathbb{R}^{d}$. 
In the case $\mathbb{X}=\mathcal{C}, \mathcal{B}(\mathcal{C})$ and $\mathcal{S}(\mathcal{C})$ are defined by the following sets:

$$
\begin{aligned}
& \mathcal{B}(\mathcal{C})=\left\{B \in \sigma(\mathcal{C}) \text { such that }\{X(0): X \in B\} \in \mathcal{B}\left(\mathbb{R}^{d}\right)\right\} \\
& \mathcal{S}(\mathcal{C})=\left\{B \in \sigma(\mathcal{C}) \text { such that }\{X(0): X \in B\} \in \mathcal{S}\left(\mathbb{R}^{d}\right)\right\}
\end{aligned}
$$

$\mathcal{M}(\mathcal{C})$ is the set of integer-valued measures on $\mathcal{C}$ such that their projection at time 0 belong to $\mathcal{M}\left(\mathbb{R}^{d}\right)$. We note $\varpi^{m}$ the Wiener measure on $\mathcal{C}$ with initial law a $\sigma$-finite measure $m$ on $\mathbb{R}^{d}$ and we substitute $\varpi^{\delta_{x}}$ by $\varpi^{x}$.

For $X \in \mathcal{C}$ and $\Gamma \in \mathcal{M}(\mathcal{C})$, we note $\operatorname{pr}_{0}(X)=X(0), \operatorname{pr}_{0}(\Gamma)=\Gamma(0)$ or $\operatorname{pr}_{0}(X, \Gamma)=(X(0), \Gamma(0))$ the projection at time 0 of $X, \Gamma$ or $(X, \Gamma)$ respectively on $\mathbb{R}^{d}, \mathcal{M}\left(\mathbb{R}^{d}\right)$ or $\mathbb{R}^{d} \times \mathcal{M}\left(\mathbb{R}^{d}\right)$. If for $t \in[0,1], \Gamma(t) \in \mathcal{M}\left(\mathbb{R}^{d}\right)$, this allows to define a projection at time $t$ denoted by $\operatorname{pr}_{t}$. Finally, for $P \in \mathcal{P}(\mathcal{M}(\mathcal{C}))$, we note $P_{0} \in \mathcal{P}\left(\mathcal{M}\left(\mathbb{R}^{d}\right)\right)$ (respectively $\mathcal{P}^{\gamma} \in \mathcal{P}(\mathcal{M}(\mathcal{C}))$ ) the probability measure $P \circ \operatorname{pr}_{0}^{-1}$ (respectively $P(\mid \Gamma(0)=\gamma)$ ).

$\left(\mathcal{F}_{t}\right)_{t \in[0,1]}$ denotes the canonical filtration respectively on $\mathcal{C}, \mathcal{M}(\mathcal{C})$ or $\mathcal{C} \times \mathcal{M}(\mathcal{C})$ generated by the appropriate projections at time $t \in[0,1]$.

\subsubsection{Point numerotation}

To identify integer-valued measures on $\mathbb{R}^{d}$ (respectively on $\mathcal{C}$ ) and sequences of distinct points on $\mathbb{R}^{d}$ (respectively on $\mathcal{C}$ ), we introduce the maps $\left(\theta_{i}\right)_{i \in \mathbb{N}^{*}}$ and $\left(\Theta_{i}\right)_{i \in \mathbb{N}^{*}}$. Let $\prec$ be the following total order on $\mathbb{R}^{d}$ - compatible with the partial order induced by the Euclidean norm - defined by: $x \prec y$ if $|x|<|y|$; if $|x|=|y|$, we compare $\frac{x}{|x|}$ and $\frac{y}{|y|}$, which belong to the unit sphere in $\mathbb{R}^{d}$. They might be represented by a sign $\varepsilon \in\{-1,+1\}$ and $d-1$ angles $\alpha_{1}, \alpha_{2}, \ldots, \alpha_{d-1} \in[0, \pi[$; so $x \prec y$, if the sign and the angles of $x$ are lower than the sign and the angles of $y$ using the lexicographic order on $\{-1,+1\} \times\left[0, \pi^{d-1}\right.$.

This point numeration is a simple case of the numerotation introduced in [23] for general polish spaces. The mesurability of such numerotation functions with respect to the natural $\sigma$-fields is dealt in [23] (Lem. 5.1.5). In our case, there is no problem.

So, for all $\gamma \in \mathcal{M}\left(\mathbb{R}^{d}\right)$, there exists a unique sequence of points $\left(\theta_{i}(\gamma)\right)_{i \in \mathbb{N}^{*}}$ in $\mathbb{R}^{d}$ such that

$$
\gamma=\sum_{i \in \mathbb{N}^{*}} \delta_{\theta_{i}(\gamma)} \text { and }\left(\theta_{i}(\gamma)\right)_{i \in \mathbb{N}^{*}} \text { is increasing in } \mathbb{R}^{d} \text { for the order } \prec \text {. }
$$

In the same way, for $\Gamma \in \mathcal{M}(\mathcal{C})$, there exists a unique sequence $\left(\Theta_{i}(\Gamma)\right)_{i \in \mathbb{N}^{*}}$ in $\mathcal{C}$ such that

$$
\Gamma=\sum_{i \in \mathbb{N}^{*}} \delta_{\Theta_{i}(\gamma)} \text { and }\left(\Theta_{i}(\Gamma)(0)\right)_{i \in \mathbb{N}^{*}} \text { is increasing in } \mathbb{R}^{d} \text { for the order } \prec
$$

We denote by $\theta$ the map from $\mathcal{M}\left(\mathbb{R}^{d}\right)$ to $\left(\mathbb{R}^{d}\right)^{\mathbb{N}^{*}}$

$$
\begin{aligned}
\theta: \mathcal{M}\left(\mathbb{R}^{d}\right) & \longrightarrow\left(\mathbb{R}^{d}\right)^{\mathbb{N}^{*}} \\
\gamma & \longmapsto\left(\theta_{i}(\gamma)\right)_{i \in \mathbb{N}^{*}},
\end{aligned}
$$

and $\Theta$ the map from $\mathcal{M}(\mathcal{C})$ to $\mathcal{C}^{\mathbb{N}^{*}}$

$$
\begin{aligned}
\Theta: \mathcal{M}(\mathcal{C}) & \longrightarrow \mathcal{C}^{\mathbb{N}^{*}} \\
\Gamma & \longmapsto\left(\Theta_{i}(\Gamma)\right)_{i \in \mathbb{N}^{*}}
\end{aligned}
$$

\subsubsection{Gibbs fields and canonical Gibbs fields}

Definition 1.1. A local Hamiltonian $H$ is a map from $\mathbb{X} \times \mathcal{M}(\mathbb{X})$ to $\mathbb{R}$ which satisfies

i) for every $X \in \mathbb{X}$, the map $\Gamma \rightarrow H(X, \Gamma)$ is $\sigma(\mathcal{M}(\mathbb{X}))$-measurable;

ii) $\forall X_{1}, X_{2} \in \mathbb{X}, \forall \Gamma \in \mathcal{M}(\mathbb{X}), H\left(X_{1}, \Gamma\right)+H\left(X_{2}, \Gamma+\delta_{X_{1}}\right)=H\left(X_{2}, \Gamma\right)+H\left(X_{1}, \Gamma+\delta_{X_{2}}\right)$. 
One often constructs local Hamiltonians by means of an interaction $\Psi$, which is defined as a measurable map from $\mathcal{F}(\mathbb{X})$, the set of finite subsets of $\mathbb{X}$, to $\mathbb{R}$. When $\Psi(\mathcal{K})=0$ for all $\mathcal{K} \in \mathcal{F}(\mathbb{X})$ such that $\operatorname{Card}(\mathcal{K}) \neq n$, then $\Psi$ is called a $n$-body interaction. If the following series converges, we construct the local Hamiltonian $H^{\Psi}$ derived from $\Psi$ by the following formula

$$
H^{\Psi}(X, \Gamma)=\sum_{\substack{\mathcal{K} \in \mathcal{F}(\mathbb{X}) \\ \mathcal{K} \subset \Gamma}} \Psi(\mathcal{K} \cup\{X\})
$$

We now define the finite volume Hamiltonian $H_{\Lambda}$, for any $\Lambda$ in $\mathcal{B}(\mathbb{X})$. For $\Gamma_{\Lambda}=\sum_{i=1}^{n} \delta_{X_{i}}$, let

$$
H_{\Lambda}\left(\Gamma_{\Lambda}, \Gamma_{\Lambda^{c}}\right)=H\left(X_{1}, \Gamma_{\Lambda^{c}}\right)+H\left(X_{2}, \Gamma_{\Lambda^{c}}+\delta_{X_{1}}\right)+\ldots+H\left(X_{n}, \Gamma_{\Lambda^{c}}+\delta_{X_{1}}+\ldots \delta_{X_{n-1}}\right)
$$

If $\mu$ is a $\sigma$-finite measure on $\mathbb{X}$ and $H$ a local Hamiltonian on $\mathbb{X}$, we define

$$
\mathcal{M}_{H, \mu}(\mathbb{X})=\bigcap_{\Lambda \in \mathcal{B}(\mathbb{X})}\left\{\Gamma \in \mathcal{M}(\mathbb{X}), \int_{\mathcal{M}(\Lambda)} \mathrm{e}^{-H_{\Lambda}\left(., \Gamma_{\Lambda^{c}}\right)} \mathrm{d} \Pi_{\Lambda}^{\mu}<+\infty\right\}
$$

Now we are able to give the definition of Gibbs fields on $\mathbb{X}$.

Definition 1.2. $\mathcal{G}(H, \mu)$, the set of Gibbs fields for the local Hamiltonian $H$ and the reference measure $\Pi^{\mu}$, is the set of probability measures $P \in \mathcal{P}(\mathcal{M}(\mathbb{X}))$ such that $P\left(\mathcal{M}_{H, \mu}(\mathbb{X})\right)=1$ and for all $\Lambda \in \mathcal{B}(\mathbb{X})$, for $P$-a.e. $\Gamma_{\Lambda^{c}}$,

$$
P\left(\mathrm{~d} \Gamma_{\Lambda} \mid \Gamma_{\Lambda^{c}}\right)=\frac{1}{Z\left(\Lambda, \Gamma_{\Lambda^{c}}\right)} \exp \left(-H_{\Lambda}\left(\Gamma_{\Lambda}, \Gamma_{\Lambda^{c}}\right)\right) \Pi_{\Lambda}^{\mu}\left(\mathrm{d} \Gamma_{\Lambda}\right),
$$

where $Z\left(\Lambda, \Gamma_{\Lambda^{c}}\right)$ is a finite normalization constant.

It is often necessary to consider mixtures of Gibbs fields. For example in Part 3, we will need canonical Gibbs fields, i.e. mixture of Gibbs fields obtained by randomizing their activity parameter. More precisely, a canonical Gibbs field is a probability measure $Q$ on $\mathcal{M}(\mathbb{X})$ verifying

$$
Q=\int_{\mathbb{R}^{+}} P^{z} \nu(\mathrm{d} z)
$$

where $P^{z}$ is a Gibbs field element of $\mathcal{G}(H, z \mu)$ and $\nu$ a probability measure on $\mathbb{R}^{+}$. We give another definition of canonical Gibbs fields, which is equivalent to the one given here (see [27]).

First, we denote by $\mathcal{M}_{H, \mu, c}(\mathbb{X})$ the following configuration set:

$$
\mathcal{M}_{H, \mu, c}(\mathbb{X})=\bigcap_{\substack{\Lambda \in \mathcal{B}(\mathbb{X}) \\ n \in \mathbb{N}}}\left\{\Gamma \in \mathcal{M}(\mathbb{X}), \int_{\mathcal{M}(\Lambda)} \mathrm{e}^{-H_{\Lambda}\left(\Gamma^{\prime}, \Gamma_{\Lambda} c\right)} \Pi_{\Lambda}^{\mu}\left(\mathrm{d} \Gamma^{\prime} \mid \Gamma^{\prime}(\Lambda)=n\right)<+\infty\right\}
$$

Definition 1.3. $\mathcal{G}_{c}(H, \mu)$, the set of canonical Gibbs fields for the local Hamiltonian $H$ and the reference measure $\Pi^{\mu}$, is the set of probability measures $P \in \mathcal{P}(\mathcal{M}(\mathbb{X}))$ such that $P\left(\mathcal{M}_{H, \mu, c}(\mathbb{X})\right)=1$ and, for every $\Lambda \in \mathcal{B}(\mathbb{X}), n \in \mathbb{N}^{*}$, for $P$-a.e. $\Gamma_{\Lambda^{c}}$,

$$
P\left(\mathrm{~d} \Gamma_{\Lambda} \mid \Gamma_{\Lambda^{c}}, \Gamma(\Lambda)=n\right)=\frac{1}{Z\left(\Lambda, n, \Gamma_{\Lambda^{c}}\right)} \mathrm{e}^{-H_{\Lambda}\left(\Gamma_{\Lambda}, \Gamma_{\Lambda^{c}}\right)} \Pi_{\Lambda}^{\mu}\left(\mathrm{d} \Gamma_{\Lambda} \mid \Gamma(\Lambda)=n\right),
$$

where $Z\left(\Lambda, n, \Gamma_{\Lambda^{c}}\right)$ is a finite normalization constant. 
We refer to $[16,27]$ for more details about Gibbs fields and canonical Gibbs fields theory.

There exists in the literature many characterizations of Gibbs fields (see for example $[1,16,28,29]$ ). In this paper, we use a characterization based on the reduced Campbell measure. Let us recall that the reduced Campbell measure $C_{P}^{!}$associated to $P \in \mathcal{P}(\mathcal{M}(\mathbb{X}))$ is the unique measure on $\mathbb{X} \times \mathcal{M}(\mathbb{X})$ such that: for every bounded measurable function from $\mathbb{X} \times \mathcal{M}(\mathbb{X})$ to $\mathbb{R}^{+}$

$$
\int_{\mathbb{X} \times \mathcal{M}(\mathbb{X})} F(X, \Gamma) C_{P}^{!}(\mathrm{d} X, \mathrm{~d} \Gamma)=\int_{\mathcal{M}(\mathbb{X})} \int_{\mathbb{X}} F\left(X, \Gamma-\delta_{X}\right) \Gamma(\mathrm{d} X) P(\mathrm{~d} \Gamma) .
$$

We now generalize to canonical Gibbs fields the characterization of Gibbs fields given in [26].

Proposition 1.1. Let $H$ be a local Hamiltonian on $\mathbb{X}$ and $\mu$ a $\sigma$-finite measure on $\mathbb{X}$. Let $P$ be a probability measure in $\mathcal{P}(\mathcal{M}(\mathbb{X}))$. If $P\left(\mathcal{M}_{H, \mu}(\mathbb{X})\right)=1$ then $P \in \mathcal{G}(H, \mu)$ if and only if $P$ satisfies

$$
C_{P}^{!}=\exp (-H) \mu \otimes P
$$

More generally, if $P\left(\mathcal{M}_{H, \mu, c}(\mathbb{X})\right)=1$ then $P \in \mathcal{G}_{c}(H, \mu)$ if and only if there exists a measure $Q$ on $\mathcal{M}(\mathbb{X})$ such that

$$
C_{P}^{!}=\exp (-H) \mu \otimes Q
$$

Moreover, if there exists a measurable map $\tilde{H}$ from $\mathbb{X} \times \mathcal{M}(\mathbb{X})$ to $\mathbb{R}$ such that $P$ satisfies

$$
C_{P}^{!}=\exp (-\tilde{H}) \mu \otimes P
$$

then $\tilde{H}$ is $\mu \otimes P$-a.s. equal to a local Hamiltonian and $P \in \mathcal{G}(\tilde{H}, \mu)$.

Proof. The first equivalence is given in [26]. To prove the equivalence between $P \in \mathcal{G}_{c}(H, \mu)$ and $(10)$, we use the representation (6) of canonical Gibbs Fields and the characterization of canonical Gibbs fields proved by Georgii in [17] (Th. 1). Finally, the last implication is proved in [18].

\subsection{Framework}

In this paper, the dimension of the space where the particles are living is equal to $d \leq 3$, except in Section 3.4 where we will present some remarks about the case $d \geq 4$.

We consider interacting Brownian particles where the interaction is induced by a symmetric pair potential $\varphi$ with compact support, of class $\mathcal{C}^{3}$ :

$$
\forall x \in \mathbb{R}^{d}, \varphi(x)=\varphi(-x) \text { and } \exists R>0 \text { such that } \varphi(x)=0 \text { for }|x|>R .
$$

Moreover, we suppose $\varphi$ superstable in the following sense: there exist $A \geq 0, B>0$ such that, for each finite sequence of points $x_{1}, x_{2}, \ldots, x_{n}$ in $\mathbb{R}^{d}$,

$$
n A+\sum_{k=1}^{n} \sum_{j \neq k} \varphi\left(x_{k}-x_{j}\right) \geq B N
$$

where $N$ is the number of pairs $\{j, k\}$ such that $\left|x_{k}-x_{j}\right| \leq R$.

The potential $\varphi$ induces a two-body interaction $\phi(\{x, y\})=\varphi(x-y)$, and a local Hamiltonian denoted by $h^{\varphi}$ and defined by:

$$
h^{\varphi}(x, \gamma)=: h^{\phi}(x, \gamma)=\sum_{y \in \gamma, y \neq x} \varphi(x-y) .
$$

Then, the interacting Brownian particle system we consider is the diffusion solution of the infinite-dimensional stochastic differential equation (1). 
If we note $\Gamma=\sum_{i \in \mathbb{N}^{*}} \delta_{X_{i}}$ and $\gamma=\sum_{i \in \mathbb{N}^{*}} \delta_{x_{i}}$, the system (1) can be written in the following way:

$$
\left\{\begin{array}{l}
\mathrm{d} \Theta_{i}(\Gamma)(t)=\mathrm{d} W_{i}(t)-\frac{1}{2} \nabla_{x} h^{\varphi}\left(\Theta_{i}(\Gamma)(t), \Gamma(t)\right) \mathrm{d} t, \forall i \in \mathbb{N}^{*} \\
\Gamma(0)=\gamma
\end{array}\right.
$$

Our paper is based on existence results proved in [12]. Fritz constructed a unique strong solution for the system (13), when $d \leq 4$ and the deterministic initial condition $\gamma$ has a finite logarithmic fluctuation energy $\mathcal{E}(\gamma)$. More precisely, let $\gamma \in \mathcal{M}\left(\mathbb{R}^{d}\right), l \in \mathbb{Z}^{d}$, and $\rho>0$. We introduce

$$
\overline{\mathcal{E}}(\gamma, l, \rho)=\sum_{x \in \gamma:|x-l| \leq \rho}\left(1+A+\sum_{y \in \gamma-\delta_{x}:|y-l| \leq \rho} \varphi(x-y)\right),
$$

where $A$ is the superstability constant appearing in (11). Remark that $\overline{\mathcal{E}}(\gamma, l, \rho)$ is non negative and it is an upper bound of the sum of the number of points of $\gamma$ in the ball $B(l, \rho)$ and the energy of $\gamma$ in this same ball. Introducing the function $g(x)=(1+\ln (1+x))^{\frac{1}{d}}$, we can define the logarithmic fluctuation energy $\mathcal{E}(\gamma)$, which has been introduced for the first time by Dobrushin and Fritz in [8]:

$$
\mathcal{E}(\gamma)=\sup _{l \in \mathbb{Z}^{d}} \sup _{r \in \mathbb{N}^{*}}\left[(r g(|l|))^{-d} \overline{\mathcal{E}}(\gamma, l, r g(|l|))+1\right] .
$$

We introduce following notations:

$$
\begin{aligned}
\mathcal{M}_{\mathcal{E}}\left(\mathbb{R}^{d}\right) & =\left\{\gamma \in \mathcal{M}\left(\mathbb{R}^{d}\right) \text { such that } \mathcal{E}(\gamma)<+\infty\right\} \\
\mathcal{M}_{\mathcal{E}}(\mathcal{C}) & =\left\{\Gamma \in \mathcal{M}(\mathcal{C}) \text { such that }\|\Gamma\|_{\mathcal{E}}=\sup _{t \in[0,1]} \mathcal{E}(\Gamma(t))<+\infty\right\} .
\end{aligned}
$$

A probability measure $\mu$ in $\mathcal{P}\left(\mathcal{M}\left(\mathbb{R}^{d}\right)\right.$ ) (respectively $P$ in $P(\mathcal{M}(\mathcal{C}))$ ) is called tempered if $\mu\left(\mathcal{M}_{\mathcal{E}}\left(\mathbb{R}^{d}\right)\right)=1$ $\left(\right.$ respectively $\left.P\left(\mathcal{M}_{\mathcal{E}}(\mathcal{C})\right)=1\right)$.

Theorem 1.1 ([12], Th. 2). If $d \leq 4$, then for every configuration $\gamma \in \mathcal{M}_{\mathcal{E}}\left(\mathbb{R}^{d}\right)$, there exists a unique strong tempered solution to (13) with initial condition $\gamma$.

Let us denote by $Q^{\gamma} \in \mathcal{P}\left(\mathcal{M}_{\mathcal{E}}(\mathcal{C})\right)$ the law of this solution. $Q^{\gamma}$ is also the unique weak solution with initial law $\delta_{\gamma}$ to the above system (15):

$$
\begin{aligned}
& \left(\Theta_{i}(\Gamma)(t)-\Theta_{i}(\Gamma)(0)+\int_{0}^{t} \frac{1}{2} \nabla \varphi * \Gamma(s)\left(\Theta_{i}(\Gamma)(s)\right) \mathrm{d} s\right)_{i \in \mathbb{N}^{*}} \\
& \text { are independant } \mathcal{F}_{t} \text {-Brownian motions in } \mathbb{R}^{d} \text { under } Q^{\gamma} \text {, }
\end{aligned}
$$

where $\frac{1}{2} \nabla \varphi * \Gamma(s)\left(\Theta_{i}(\Gamma)(s)\right)$ denotes the convolution of the function $\frac{1}{2} \nabla \varphi$ by the measure $\Gamma(s)$, evaluated at the point $\Theta_{i}(\Gamma)(s)$.

Obviously, the solution of the above system (15) is Markovian so that the probability measure $Q^{\mu}=$ $\int_{\mathcal{M}_{\mathcal{E}\left(\mathbb{R}^{d}\right)}} Q^{\gamma} \mu(\mathrm{d} \gamma)$ is the unique solution of system (15) with initial law $\mu$.

Systems with more general interaction $\varphi$ have been considered for exemple in [11,33], but their construction is always done in a stationary context. 


\section{Interacting Brownian particle system and associated Gibbs fields on $\mathcal{C}$}

In the following proposition, we recall an estimate due to Fritz on the logarithmic fluctuation of any solution of system (15).

Proposition 2.1 ([12], Prop. 2). Let $d \leq 3$; then, for all $\varepsilon \in] 0,1[$, there exist two strictly positive constants a and $b$ such that, for every $\gamma \in \mathcal{M}_{\mathcal{E}}\left(\mathbb{R}^{d}\right)$ and $u>0$, the following inequality holds:

$$
Q^{\gamma}\left(\|\Gamma\|_{\mathcal{E}}>u\right) \leq a \exp \left(-\frac{b}{\mathcal{E}(\gamma)^{2}} u^{1-\epsilon}\right)
$$

\subsection{Regularity results for interacting Brownian particles}

2.1.1. Uniform bound $\zeta$ of the weighted fluctuation of each particle around its initial position

For $\Gamma \in \mathcal{M}(\mathcal{C})$, we denote $X_{i}=\Theta_{i}(\Gamma)$ and $x_{i}=\theta_{i}(\gamma)$. Let $\left.\eta \in\right] 0,1[$ be fixed in this Section 2.1. We study the following random variable

$$
\zeta(\Gamma)=\sup _{k \in \mathbb{N}^{*}} \sup _{0 \leq t \leq 1} \frac{\left|X_{k}(t)-X_{k}(0)\right|}{\left(1+\left|X_{k}(0)\right|\right)^{\eta}} .
$$

In [14], the author proved that $Q^{\gamma}$-a.s., $\zeta<+\infty$. We need here a more precise estimate of the law of $\zeta$.

Proposition 2.2. For all $\varepsilon>0$, there exist two strictly positive constants $a_{1}$ and $b_{1}$ such that, for every $\gamma \in \mathcal{M}_{\mathcal{E}}\left(\mathbb{R}^{d}\right)$ and $u>0$, the following inequality holds:

$$
Q^{\gamma}(\zeta \geq u) \leq a_{1} \mathcal{E}(\gamma)^{2} \exp \left(-\frac{b_{1}}{\mathcal{E}(\gamma)^{2}} u^{1-\varepsilon}\right)
$$

In particular, $\zeta$ is finite $Q^{\gamma}$-almost surely and admits moments of all orders under $Q^{\gamma}$.

Proof. To begin, we enounce some easy properties of any point measures in $\mathcal{M}_{\mathcal{E}}(\mathcal{C})$.

Lemma 2.1. Let $\Gamma \in \mathcal{M}_{\mathcal{E}}(\mathcal{C}) ; \Gamma$ satisfies both following inequalities:

$$
\begin{gathered}
\forall n \in \mathbb{N}^{*}, \quad \sup _{t \in[0,1]} \Gamma(t)(B(0, n)) \leq n^{d}\|\Gamma\|_{\mathcal{E}}, \\
\forall t \in[0,1], \forall k \geq 2, \quad \theta_{k}(\Gamma(t)) \geq\left(\frac{k-1}{\mathcal{E}(\Gamma(t))}\right)^{\frac{1}{d}} .
\end{gathered}
$$

For all $t \in[0,1]$ and $k \in \mathbb{N}^{*}$, we denote by $\bar{N}(t, k)(\Gamma)$ the random variable $\Gamma(t)\left(B\left(X_{k}(t), R\right)\right)$, that is the cardinality of particles which are at time $t$ in the ball centered at $X_{k}(t)$ with radius $R$; let also define

$$
N(\Gamma)=1+\sup _{k \in \mathbb{N}^{*}} \sup _{0 \leq t \leq 1} \frac{\bar{N}(t, k)}{\left(1+\left|X_{k}(t)\right|\right)^{\eta}} .
$$

Lemma 2.2. For all $\varepsilon \in] 0,1\left[\right.$, there exists two strictly positive constants $a_{2}$ and $b_{2}$ such that for all $\gamma \in \mathcal{M}_{\mathcal{E}}\left(\mathbb{R}^{d}\right)$ and $u>0$, we have

In particular, $N$ is finite $Q^{\gamma}$-almost surely.

$$
Q^{\gamma}(N \geq u) \leq a_{2} \exp \left(-\frac{b_{2}}{\mathcal{E}(\gamma)^{2}} u^{1-\epsilon}\right)
$$

Proof. Let $R^{\prime}=\mathrm{e}^{\left((R+\sqrt{2})^{d}-1\right)}-1$; so, for all $x \in \mathbb{R}^{d}$ such that $|x| \geq R^{\prime}$,

$$
\text { there exists } l \in \mathbb{Z}^{d} \text { such that }|x-l|<\sqrt{2} \text { and } B(x, R) \subset B(l, g(|l|)) \text {. }
$$


Let $t \in[0,1]$ and $k \in \mathbb{N}^{*}$.

If $\left|X_{k}(t)\right| \leq R^{\prime}$, then $B\left(X_{k}(t), R\right) \subset B\left(0, R^{\prime}+R\right)$. Using Lemma 2.1 we obtain

$$
\bar{N}(t, k) \leq\|\Gamma\|_{\mathcal{E}}\left(R^{\prime}+R+1\right)^{d} .
$$

If $\left|X_{k}(t)\right|>R^{\prime}$, then by (17) there exists a point $l$ in $\mathbb{Z}^{d}$ such that $B\left(X_{k}(t), R\right) \subset B(l, g(|l|))$ and, due to Lemma 2.1, we deduce

$$
\bar{N}(t, k) \leq g(|l|)^{d}\|\Gamma\|_{\mathcal{E}} \leq g\left(\left|X_{k}(t)\right|+\sqrt{2}\right)^{d}\|\Gamma\|_{\mathcal{E}}
$$

So,

$$
N \leq 1+\|\Gamma\|_{\mathcal{E}} \sup _{k \in \mathbb{N}^{*}} \sup _{0 \leq t \leq 1} \max \left(\frac{g\left(\left|X_{k}(t)\right|+\sqrt{2}\right)^{d}}{\left(1+\left|X_{k}(t)\right|\right)^{\eta}},\left(R^{\prime}+R+1\right)^{d}\right) ;
$$

the function $\frac{g(x+\sqrt{2})^{d}}{(1+x)^{\eta}}$ being bounded, and using also Proposition 2.1, we easily obtain the desired estimate for the tail of the law of $N$ under $Q^{\gamma}$.

We denote by $\left(B_{k}\right)_{k \in \mathbb{N}^{*}}$ the processes defined by

$$
B_{k}(t)=X_{k}(t)-X_{k}(0)+\frac{1}{2} \int_{0}^{t} \sum_{i \neq k} \nabla \varphi\left(X_{k}(s)-X_{i}(s)\right) \mathrm{d} s .
$$

It is a family of independent Brownian motions under $Q^{\gamma}$.

For $k \in \mathbb{N}^{*}$, we have

$$
\left|X_{k}(t)-X_{k}(0)\right| \leq \int_{0}^{t} \sum_{i \neq k} \frac{1}{2}\left|\nabla \varphi\left(X_{k}(s)-X_{i}(s)\right)\right| \mathrm{d} s+\left|B_{k}(t)\right| .
$$

So,

$$
\left|X_{k}(t)-X_{k}(0)\right| \leq\left(\frac{1}{2}\|\nabla \varphi\|_{\infty}+1\right) N \int_{0}^{t}\left(1+X_{k}(s)\right)^{\eta} \mathrm{d} s+\left|B_{k}(t)\right| .
$$

Let us define $\bar{X}_{k}=\sup _{0 \leq t \leq 1}\left|X_{k}(t)-X_{k}(0)\right| ;$ then

$$
\bar{X}_{k} \leq \xi\left(\left(1+\left|X_{k}(0)\right|\right)^{\eta}+\left(1+\bar{X}_{k}\right)^{\eta}\right)
$$

where $\xi$ is the following random variable

$$
\xi=\left(\frac{1}{2}\|\nabla \varphi\|_{\infty}+1\right)\left(N+\sup _{k \in \mathbb{N}^{*}} \sup _{0 \leq t \leq 1} \frac{\left|B_{k}(t)\right|}{\left(1+\left|X_{k}(0)\right|\right)^{\eta}}\right) .
$$

Lemma 2.3. For all $\varepsilon>0$, there exists two strictly positive constants $a_{3}$ and $b_{3}$, such that for all $\gamma \in \mathcal{M}_{\mathcal{E}}\left(\mathbb{R}^{d}\right)$ and $u>0$, the following inequality holds:

$$
Q^{\gamma}(\xi \geq u) \leq a_{3} \mathcal{E}(\gamma)^{2} \exp \left(-\frac{b_{3}}{\mathcal{E}(\gamma)^{2}} u^{1-\epsilon}\right)
$$

in particular, $\xi$ is finite $Q^{\gamma}$-almost surely.

Proof. Due to Lemma 2.2, it is sufficient to control the tail of the law of the random variable

$$
\sup _{k \in \mathbb{N}^{*}} \sup _{0 \leq t \leq 1} \frac{\left|B_{k}(t)\right|}{\left(1+\left|X_{k}(0)\right|\right)^{\eta}} .
$$


Let $\varepsilon>0$ and $\gamma \in \mathcal{M}_{\mathcal{E}}\left(\mathbb{R}^{d}\right)$; since one knows explicitely the law of the supremum of the Brownian motion, there exists a constant $C_{1}>0$ such that, for all $u \geq 1$,

$$
Q^{\gamma}\left(\sup _{0 \leq t \leq 1}\left|B_{k}(t)\right| \geq u\left(1+\left|X_{k}(0)\right|\right)^{\eta}\right) \leq C_{1} \mathrm{e}^{-u^{2} / 4} \exp \left(-\frac{1}{4}\left(1+\left|X_{k}(0)\right|\right)^{2 \eta}\right) .
$$

Remark that $\sum_{k \in \mathbb{N}^{*}} \exp \left(-\frac{1}{4}\left(1+\left|X_{k}(0)\right|\right)^{2 \eta}\right)$ is finite and let us compute an upper bound of this sum as a function of $\mathcal{E}(\gamma)$ : there exists a constant $C_{2}>0$ such that, for all $x \geq C_{2},(1+x)^{2 \eta} \geq 8 d \ln (x)$. So, using Lemma 2.1, we obtain for $k \geq k_{0}=\mathcal{E}(\gamma) C_{2}^{d}+1$

$$
\exp \left(-\frac{1}{4}\left(1+\left|X_{k}(0)\right|\right)^{2 \eta}\right) \leq\left(\frac{k-1}{\mathcal{E}(\gamma)}\right)^{-2} .
$$

Thus, there exists a constant $C_{3}>0$ such that

$$
\begin{aligned}
\sum_{k \geq 1} \exp \left(-\frac{1}{4}\left(1+\left|X_{k}(0)\right|\right)^{2 \eta}\right) & \leq \mathcal{E}(\gamma) C_{2}^{d}+\sum_{k \geq k_{0}} \exp \left(-\frac{1}{4}\left(1+\left|X_{k}(0)\right|\right)^{2 \eta}\right) \\
& \leq C_{3} \mathcal{E}(\gamma)^{2} .
\end{aligned}
$$

So, there exists a constant $C_{4}>0$ such that for all $u>0$,

$$
Q^{\gamma}\left(\sup _{k \in \mathbb{N}^{*}} \sup _{0 \leq t \leq 1} \frac{\left|B_{k}(t)\right|}{\left(1+\left|X_{k}(0)\right|\right)^{\eta}} \geq u\right) \leq C_{4} \mathcal{E}(\gamma)^{2} \mathrm{e}^{-\frac{1}{4} u} .
$$

We can now complete the proof of Proposition 2.2. Consider the inequality (18)

$$
\bar{X}_{k}-\xi\left(1+\bar{X}_{k}\right)^{\eta} \leq \xi\left(1+\left|X_{k}(0)\right|\right)^{\eta} .
$$

To solve this inequality, we introduce the functions $\left(h_{\tau}\right)_{\tau \in \mathbb{R}^{+}}$defined by $h_{\tau}(x)=x-\tau(1+x)^{\eta}$. A elementary study of the function $h_{\tau}(x)-\frac{1}{2} x$ proves that for all $x>0$

$$
h_{\tau}(x) \geq \frac{1}{2} x-1-C_{5}(\eta) \tau^{\frac{1}{1-\eta}},
$$

where $C_{5}(\eta)$ is a positive constant which depends only on $\eta$. We deduce from (18) and (20) that there exists a constant $C_{6}(\eta)$ such that

$$
\bar{X}_{k} \leq C_{6}(\eta) \xi^{\frac{1}{1-\eta}}\left(1+\left|X_{k}(0)\right|\right)^{\eta} .
$$

Therefore, let $\varepsilon>0, \gamma \in \mathcal{M}_{\mathcal{E}}\left(\mathbb{R}^{d}\right)$ and $\eta^{\prime}$ a positive constant such that $\eta^{\prime}<\min (\varepsilon, \eta)$; on account of $(21)$ we have

$$
\begin{aligned}
Q^{\gamma}\left(\sup _{k \in \mathbb{N}^{*}} \sup _{0 \leq t \leq 1} \frac{\left|X_{k}(t)-X_{k}(0)\right|}{\left(1+\left|X_{k}(0)\right|\right)^{\eta}} \geq u\right) & \leq Q^{\gamma}\left(\sup _{k \in \mathbb{N}^{*}} \sup _{0 \leq t \leq 1} \frac{\left|X_{k}(t)-X_{k}(0)\right|}{\left(1+\left|X_{k}(0)\right|\right)^{\eta^{\prime}}} \geq u\right) \\
& \leq Q^{\gamma}\left(\xi \geq\left(\frac{u}{C_{6}\left(\eta^{\prime}\right)}\right)^{1-\eta^{\prime}}\right) \\
& \leq a_{3}^{\prime} \mathcal{E}(\gamma)^{2} \exp \left(-\frac{b_{3}^{\prime}}{\mathcal{E}(\gamma)^{2} C_{6}\left(\eta^{\prime}\right)^{\left(1-\eta^{\prime}\right)\left(1-\varepsilon^{\prime}\right)}} u^{\left(1-\eta^{\prime}\right)\left(1-\varepsilon^{\prime}\right)}\right),
\end{aligned}
$$

where $\varepsilon^{\prime}>0$ is chosen such that $\left(1-\eta^{\prime}\right)\left(1-\varepsilon^{\prime}\right)=(1-\varepsilon)$ and $a_{3}^{\prime}, b_{3}^{\prime}$ are the constants associated to $\varepsilon^{\prime}$ in Lemma 2.3. Inequality (16) is thus proven. 
2.1.2. Estimate of the number of particles interacting with a fixed particle

We first give an estimate of the initial position of particles which, at some time $t \in[0,1]$, may come into the ball $B\left(X_{i_{0}}(t), 2 R\right)$, where $i_{0}$ is a integer, fixed along Section 2.1.2.

Lemma 2.4. One can construct two non decreasing functions $K_{1}$ and $K_{2}$ from $\mathbb{R}^{+}$to $\mathbb{R}^{+}$such that, for every $\Gamma$ satisfying $\zeta(\Gamma)<+\infty$, as soon as there exists $i \in \mathbb{N}^{*}$ and $t \in[0,1]$ such that $\left|X_{i}(t)-X_{i_{0}}(t)\right| \leq 2 R$, then

$$
\left|X_{i}(0)\right| \leq K_{1}\left(\left|X_{i_{0}}\right|\right)+K_{2}\left(\left|X_{i_{0}}\right|\right) \zeta^{\frac{1}{1-\eta}} .
$$

Proof. Let $i \in \mathbb{N}^{*}$ and $t \in[0,1]$ such that $\left|X_{i}(t)-X_{i_{0}}(t)\right| \leq 2 R$; then

$$
\begin{aligned}
\left|x_{i}\right| & \leq\left|X_{i}(t)-x_{i}\right|+\left|X_{i}(t)-X_{i_{0}}(t)\right|+\left|X_{i_{0}}(t)-x_{i_{0}}\right|+\left|x_{i_{0}}\right| \\
& \leq \zeta\left(1+\left|x_{i}\right|\right)^{\eta}+2 R+\zeta\left(1+\left|x_{i_{0}}\right|\right)^{\eta}+\left|x_{i_{0}}\right|
\end{aligned}
$$

which implies that

$$
\left|x_{i}\right|-\zeta\left(1+\left|x_{i}\right|\right)^{\eta} \leq \zeta\left(1+\left|x_{i_{0}}\right|\right)^{\eta}+2 R+\left|x_{i_{0}}\right|
$$

Using inequality (20), it is easy to deduce (22).

Let $\beta$ be the map from $\mathbb{R}^{+} \times \mathcal{M}\left(\mathbb{R}^{d}\right) \times \mathbb{N}^{*}$ to $\mathbb{N}$ defined by

$$
\beta(z, \gamma, i)=\gamma\left(B\left(0, K_{1}\left(\left|x_{i}\right|\right)+K_{2}\left(\left|x_{i}\right|\right) z^{\frac{1}{1-\eta}}\right)\right) .
$$

For every $\Gamma$ such that $\zeta(\Gamma)<+\infty$ and $\Gamma(0)=\gamma, \beta\left(\zeta, \gamma, i_{0}\right)$ is an upper bound of the number of particles which could come at some time $t \in[0,1]$ at distance smaller than $2 \mathrm{R}$ from the particle $i_{0}$. We now prove some estimates for the random variable $\beta$.

Lemma 2.5. There exists two functions $K_{3}$ and $K_{4}$ from $\mathcal{M}_{\mathcal{E}}\left(\mathbb{R}^{d}\right) \times \mathbb{N}^{*}$ to $\mathbb{R}^{+}$such that

$$
\forall z \in \mathbb{R}^{+}, \forall \gamma \in \mathcal{M}_{\mathcal{E}}\left(\mathbb{R}^{d}\right), \forall i \in \mathbb{N}^{*}, \quad \beta(z, \gamma, i) \leq K_{3}(\gamma, i)+K_{4}(\gamma, i) z^{\frac{d}{1-\eta}} .
$$

Moreover, for all $\gamma, \gamma^{\prime} \in \mathcal{M}_{\mathcal{E}}\left(\mathbb{R}^{d}\right)$ such that $\mathcal{E}(\gamma) \leq \mathcal{E}\left(\gamma^{\prime}\right)$ and for some $i \in \mathbb{R}^{+},\left|\theta_{i}(\gamma)\right| \leq\left|\theta_{i}\left(\gamma^{\prime}\right)\right|$ then

$$
K_{3}(\gamma, i) \leq K_{3}\left(\gamma^{\prime}, i\right) \text { and } K_{4}(\gamma, i) \leq K_{4}\left(\gamma^{\prime}, i\right) .
$$

Proof. By Lemma 2.1, for every $\gamma \in \mathcal{M}_{\mathcal{E}}\left(\mathbb{R}^{d}\right)$, the number of particles in the ball $B\left(0, K_{1}\left(\left|x_{i}\right|\right)+K_{2}\left(\left|x_{i}\right|\right) z^{\frac{1}{1-\eta}}\right)$ is bounded by $\left(K_{1}\left(\left|x_{i}\right|\right)+K_{2}\left(\left|x_{i}\right|\right) z^{\frac{1}{1-\eta}}\right)^{d} \mathcal{E}(\gamma)$. So, using Lemma 2.4 , we easily prove the estimates for $\beta$.

\subsection{Interacting Brownian particles as Gibbs fields on $\mathcal{C}$}

The aim of this section is to prove that solutions of system (15) with a tempered Gibbs field as initial law, are Gibbs fields on $\mathcal{C}(c f$. Th. 2.1). In the following proposition, we exhibit a large set of tempered Gibbs fields on $\mathbb{R}^{d}$ by providing an estimate for the law of the logarithmic fluctuation energy under these Gibbs fields. For a related result, see [13] too. It will be used in Section 2.3.

Proposition 2.3. Let $\mu$ be a Gibbs field in $\mathcal{G}\left(h^{\psi}, \lambda\right)$ where $\psi$ is a multi-body superstable and lower regular interaction. Then there exists two strictly positive constants $a_{4}$ and $b_{4}$ such that for all $u>0$

$$
\mu(\mathcal{E}(\gamma) \geq u) \leq a_{4} \mathrm{e}^{-b_{4} u}
$$

In particular, $\mu$ is tempered.

For the exact definition of multi-body superstable and lower regular interaction, we refer to [32] (p. 128). 
Proof. For $k=\left(k_{1}, \ldots, k_{d}\right) \in \mathbb{Z}^{d}$, we denote by $D_{k}$ the following cube in $\left.\left.\left.\left.\mathbb{R}^{d},\right] k_{1}-\frac{1}{2}, k_{1}+\frac{1}{2}\right] \times \ldots\right] k_{d}-\frac{1}{2}, k_{d}+\frac{1}{2}\right]$. For every $l \in \mathbb{Z}^{d}$ and $\rho \geq 1, E_{l, \rho}$ is the minimal subset of $\mathbb{Z}^{d}$ such that

$$
B(l, \rho) \subset \bigcup_{k \in E_{l, \rho}} D_{k}
$$

By definition of $\overline{\mathcal{E}}(\gamma, l, \rho)$ and using the fact that $\varphi$ has a compact support, we deduce that there exists a constant $C_{0}$ such that

$$
\overline{\mathcal{E}}(\gamma, l, \rho) \leq C_{0} \sum_{k \in E_{l, \rho}} \gamma\left(D_{k}\right)^{2}
$$

By Corollary 2.8 in [32], there exists two constants $C_{1}$ and $C_{2}$ such that, for all $u \geq 0$,

$$
\mu\left(\frac{\overline{\mathcal{E}}(\gamma, l, \rho)}{\rho^{d}} \geq u\right) \leq \mathrm{e}^{\rho^{d}\left(C_{2}-C_{1} u\right)}
$$

Now, let us analyse the tail of the law of $\mathcal{E}(\gamma)$.

$$
\begin{aligned}
\mathcal{E}(\gamma) & =\sup _{l \in \mathbb{Z}^{d}} \sup _{r \in \mathbb{N}^{*}}\left((r g(|l|))^{-d} \overline{\mathcal{E}}(\gamma, l, r g(|l|))+1\right) \\
& =\sup _{n \in \mathbb{N}^{*}}\left(\rho_{n}^{-d} \overline{\mathcal{E}}\left(\gamma, l_{n}, \rho_{n}\right)+1\right)
\end{aligned}
$$

where the sequence $\left(l_{n}, \rho_{n}\right)_{n \in \mathbb{N}^{*}}$ is constructed in such a way that the following sets coincide

$$
\left\{B(l, r g(|l|)), l \in \mathbb{Z}^{d}, r \in \mathbb{N}^{*}\right\}=\left\{B\left(l_{n}, \rho_{n}\right), n \in \mathbb{N}^{*}\right\}
$$

and $\left(\rho_{n}\right)_{n \in \mathbb{N}^{*}}$ is increasing.

Let us compute a lower bound for $\rho_{n}$. Let $n \in \mathbb{N}^{*}$, we have

$\left\{B(l, r g(|l|)), l \in \mathbb{Z}^{d} r \in \mathbb{N}^{*}\right.$ such that $\left.r g(|l|) \leq \rho_{n}\right\} \subset \bigcup_{1 \leq r \leq \rho_{n}}\left\{B(l, r g(|l|)), l \in \mathbb{Z}^{d}\right.$ such that $\left.|l| \leq g^{*}\left(\rho_{n}\right)\right\}$,

where $g^{*}$ is the inverse function of $g$. So there exists $C_{3}>0$ such that, for every $n \in \mathbb{N}^{*}$

$$
\operatorname{Card}\left\{B(l, r g(|l|)), l \in \mathbb{Z}^{d} r \in \mathbb{N}^{*} \text { such that } r g(|l|) \leq \rho_{n}\right\} \leq C_{3} g^{*}\left(\rho_{n}\right)^{d+1} .
$$

Since $C_{3} g^{*}\left(\rho_{n}\right)^{d+1}$ is greater than $n$ there exists some constants $C_{4}>0$ and $C_{5}>0$ such that

$$
\rho_{n} \geq C_{4} \ln \left(C_{5} n\right)^{\frac{1}{d}}
$$


From (24) and (25), we deduce that for $u \geq \frac{C_{2}}{C_{1}}+\frac{2}{C_{1} C_{4}^{d}}$

$$
\begin{aligned}
\sum_{n \geq 1} \mu\left(\frac{\overline{\mathcal{E}}\left(\gamma, l_{n}, \rho_{n}\right)}{\rho_{n}^{d}} \geq u\right) & \leq \sum_{1 \leq n \leq \frac{1}{C_{5}}} \mathrm{e}^{C_{2}-C_{1} u}+\sum_{n \geq\left[\frac{1}{C_{5}}\right]+1} \mathrm{e}^{C_{4}^{d} \ln \left(C_{5} n\right)\left(C_{2}-C_{1} u\right)} \\
& \leq \frac{\mathrm{e}^{C_{2}}}{C_{5}} \mathrm{e}^{-C_{1} u}+\sum_{n \geq\left[\frac{1}{C_{5}}\right]+1}\left(C_{5} n\right)^{C_{4}^{d}\left(C_{2}-C_{1} u\right)} \\
& \leq \frac{\mathrm{e}^{C_{2}}}{C_{5}} \mathrm{e}^{-C_{1} u}+\left(C_{5}\left(\left[\frac{1}{C_{5}}\right]+1\right)\right)^{C_{4}^{d}\left(C_{2}-C_{1} u\right)} \sum_{n \geq\left[\frac{1}{C_{5}}\right]+1}\left(\frac{n}{\left[\frac{1}{C_{5}}\right]+1}\right)^{-2} \\
& \leq a_{4} \mathrm{e}^{-b_{4} u},
\end{aligned}
$$

where $a_{4}>0$ and $b_{4}>0$ are constants. Therefore, for $u$ sufficiently large, $\mu(\mathcal{E}(\gamma) \geq u) \leq a_{4} \mathrm{e}^{-b_{4} u}$; adjusting the constants $a_{4}$ and $b_{4}$ this inequality holds also for all $u>0$.

Coming back to the aim of this section, let us define a functional which will be the local dynamical Hamiltonian on the pathspace in the main Theorem 2.1. For $X \in \mathcal{C}$ and $\Gamma \in \mathcal{M}(\mathcal{C})$,

$$
\begin{aligned}
H^{\Phi}(X, \Gamma)= & \frac{1}{2} \sum_{Y \in \Gamma \backslash X}(\varphi(X(1)-Y(1))-\varphi(X(0)-Y(0)) \\
& \left.-\int_{0}^{1}\left(\Delta \varphi-\frac{1}{2}|\nabla \varphi|^{2}\right)(X(s)-Y(s)) \mathrm{d} s\right) \\
& +\frac{1}{4} \sum_{\{Y, Z\} \subset \Gamma \backslash X} \int_{0}^{1}(\nabla \varphi(X(s)-Y(s)) \cdot \nabla \varphi(X(s)-Z(s)) \\
& +\nabla \varphi(Y(s)-X(s)) \cdot \nabla \varphi(Y(s)-Z(s)) \\
& +\nabla \varphi(Z(s)-X(s)) \cdot \nabla \varphi(Z(s)-Y(s))) \mathrm{d} s .
\end{aligned}
$$

Remark that $H^{\Phi}$ is associated to the following two-body and three-body interaction $\Phi$ :

$$
\begin{array}{cl}
\Phi(\{X, Y\})= & \frac{1}{2}(\varphi(X(1)-Y(1))-\varphi(X(0)-Y(0)) \\
& \left.-\int_{0}^{1}\left(\Delta \varphi-\frac{1}{2}|\nabla \varphi|^{2}\right)(X(s)-Y(s)) \mathrm{d} s\right) \\
\Phi(\{X, Y, Z\})= & \frac{1}{4} \int_{0}^{1}[\nabla \varphi(X(s)-Y(s)) \cdot \nabla \varphi(X(s)-Z(s)) \\
& +\nabla \varphi(Y(s)-X(s)) \cdot \nabla \varphi(Y(s)-Z(s)) \\
& +\nabla \varphi(Z(s)-X(s)) \cdot \nabla \varphi(Z(s)-Y(s))] \mathrm{d} s . \\
\Phi(\mathcal{K})= & 0 \quad \text { if } \operatorname{Card}(\mathcal{K}) \notin\{2,3\} .
\end{array}
$$


Furthermore, $H^{\Phi}(X, \Gamma)$ is not defined for all $X \in \mathcal{C}$ and $\Gamma \in \mathcal{M}(\mathcal{C})$, but due to Lemma 2.4 the formula (26) has a sense $Q^{\gamma}$-almost surely (the sums are finite $Q^{\gamma}$-a.s.).

Theorem 2.1. Let $h$ be a local Hamiltonian on $\mathbb{R}^{d}$ and $m$ a $\sigma$-finite measure on $\mathbb{R}^{d}$; then the following assertions hold:

$$
\begin{aligned}
& \text { for each tempered } \mu \in \mathcal{G}(h, m), Q^{\mu} \in \mathcal{G}\left(h \circ \mathrm{pr}_{0}+H^{\Phi}, \varpi^{m}\right), \\
& \text { for each tempered } \mu \in \mathcal{G}_{c}(h, m), Q^{\mu} \in \mathcal{G}_{c}\left(h \circ \mathrm{pr}_{0}+H^{\Phi}, \varpi^{m}\right) .
\end{aligned}
$$

Before giving the proof of the above theorem, let us show that in the following sense:

Lemma 2.6. Let $\gamma \in \mathcal{M}_{\mathcal{E}}\left(\mathbb{R}^{d}\right) ;$ for any $i_{0} \in \mathbb{N}^{*}$ and all measurable bounded functional $F$ from $\mathcal{C} \times \mathcal{M}(\mathcal{C})$ to $\mathbb{R}$ we have

$$
\int_{\mathcal{M}(\mathcal{C})} \exp \left(H^{\Phi}\left(\Theta_{i_{0}}(\Gamma), \Gamma\right)\right) F\left(\Theta_{i_{0}}(\Gamma), \Gamma-\delta_{\Theta_{i_{0}}(\Gamma)}\right) Q^{\gamma}(\mathrm{d} \Gamma)=\int_{\mathcal{M}(\mathcal{C})} \int_{\mathcal{C}} F(X, \Gamma) Q^{\gamma-\delta_{\theta_{i_{0}}}(\gamma)} \otimes \varpi^{\theta_{i_{0}}(\gamma)}(\mathrm{d} \Gamma, \mathrm{d} X)
$$

Proof. Another way to write $H^{\Phi}$ is the following:

$$
\begin{aligned}
H^{\Phi}\left(X_{i_{0}}, \Gamma\right)= & \frac{1}{2} \sum_{i \neq i_{0}}\left(\varphi\left(X_{i_{0}}(1)-X_{i}(1)\right)-\varphi\left(X_{i_{0}}(0)-X_{i}(0)\right)-\int_{0}^{1} \Delta \varphi\left(X_{i_{0}}(s)-X_{i}(s)\right) \mathrm{d} s\right) \\
& -\sum_{i \neq i_{0}} \frac{1}{2} \int_{0}^{1} \nabla \varphi\left(X_{i_{0}}(s)-X_{i}(s)\right) \cdot \sum_{j \neq i} \frac{1}{2} \nabla \varphi\left(X_{i}(s)-X_{j}(s)\right) \mathrm{d} s \\
& +\frac{1}{2} \int_{0}^{1} \frac{1}{4}\left|\sum_{i \neq i_{0}} \nabla \varphi\left(X_{i_{0}}(s)-X_{i}(s)\right)\right|^{2} \mathrm{~d} s \\
& -\frac{1}{2} \sum_{i \neq i_{0}} \int_{0}^{1} \frac{1}{4}\left|\nabla \varphi\left(X_{i_{0}}(s)-X_{i}(s)\right)\right|^{2} \mathrm{~d} s .
\end{aligned}
$$

Since the process

$$
\left(X_{i}(t)-X_{i}(0)+\int_{0}^{t} \frac{1}{2} \sum_{j \neq i} \nabla \varphi\left(X_{i}(s)-X_{j}(s)\right) \mathrm{d} s\right)_{i \in \mathbb{N}^{*}}
$$

which we denote by $\left(B_{i}\right)_{i \in_{\mathbb{N}^{*}}}$ is an infinite family of independent Brownian motions under $Q^{\gamma}$, we can use Ito's formula to obtain for each $i \neq i_{0}$

$$
\begin{aligned}
\varphi\left(X_{i_{0}}(1)-X_{i}(1)\right)-\varphi\left(X_{i_{0}}(0)-X_{i}(0)\right) \\
\quad=\int_{0}^{1} \nabla \varphi\left(X_{i_{0}}(s)-X_{i}(s)\right) \cdot\left(\mathrm{d} X_{i_{0}}(s)-\mathrm{d} X_{i}(s)\right)+\int_{0}^{1} \Delta \varphi\left(X_{i_{0}}(s)-X_{i}(s)\right) \mathrm{d} s .
\end{aligned}
$$


Thus, together with Lemma 2.5 this implies that

$$
\begin{aligned}
H^{\Phi}\left(X_{i_{0}}, \Gamma\right)= & \int_{0}^{1} \frac{1}{2} \sum_{\substack{i \neq i_{0} \\
1 \leq i \leq \beta\left(\zeta, \gamma, i_{0}\right)}} \nabla \varphi\left(X_{i_{0}}(s)-X_{i}(s)\right) \mathrm{d} B_{i_{0}}(s) \\
& -\frac{1}{2} \int_{0}^{1}\left|\frac{1}{2} \sum_{\substack{i \neq i_{0} \\
1 \leq i \leq \beta\left(\zeta, \gamma, i_{0}\right)}} \nabla \varphi\left(X_{i_{0}}(s)-X_{i}(s)\right)\right|^{2} \mathrm{~d} s \\
& +\sum_{\substack{i \neq i_{0} \\
1 \leq i \leq \beta\left(\zeta, \gamma, i_{0}\right)}} \int_{0}^{1}-\frac{1}{2} \nabla \varphi\left(X_{i_{0}}(s)-X_{i}(s)\right) \mathrm{d} B_{i}(s) \\
& -\frac{1}{2} \sum_{\substack{i \neq i_{0} \\
1 \leq i \leq \beta\left(\zeta, \gamma, i_{0}\right)}} \int_{0}^{1}\left|\frac{1}{2} \nabla \varphi\left(X_{i_{0}}(s)-X_{i}(s)\right)\right|^{2} \mathrm{~d} s
\end{aligned}
$$

Therefore $\exp \left(H^{\Phi}\left(X_{i_{0}}, \Gamma\right)\right)$ is equal to an exponential local martingale evaluated at time 1:

$$
\exp \left(H^{\Phi}\left(X_{i_{0}}, \Gamma\right)\right)=\exp \left(\sum_{i=1}^{+\infty} \int_{0}^{1} A_{i}(s) \mathrm{d} B_{i}(s)-\frac{1}{2} \int_{0}^{1}\left|A_{i}(s)\right|^{2} \mathrm{~d} s\right)
$$

where

$$
A_{i}(s)= \begin{cases}\frac{1}{2} \sum_{\substack{j \neq i_{0} \\ 1 \leq j \leq \beta\left(\zeta, \gamma, i_{0}\right)}} \nabla \varphi\left(X_{i_{0}}(s)-X_{j}(s)\right) & \text { si } i=i_{0} \\ \frac{1}{2} \nabla \varphi\left(X_{i}(s)-X_{i_{0}}(s)\right) & \text { si } i \neq i_{0} .\end{cases}
$$

Thanks to Proposition 2.2, $\zeta$ is $Q^{\gamma}$-almost surely finite and then it is $Q^{\gamma-\delta_{x_{0}}} \otimes \varpi^{x_{i_{0}}}$-almost surely finite too; this implies

$$
\sum_{i} \int_{0}^{1}\left|A_{i}(t)\right|^{2} \mathrm{~d} t<+\infty \quad Q^{\gamma-\delta_{x_{0}}} \otimes \varpi^{x_{i_{0}}}-\text { p.s. }
$$

From the uniqueness of tempered solutions of system (15) for $\mu=\delta_{\gamma}$ and $\mu=\delta_{\gamma-\delta_{x_{i}}}$ we deduce the absolute continuity of $Q^{\gamma-\delta_{x_{0}}} \otimes \varpi^{x_{i_{0}}}$ with respect to $Q^{\gamma}$ (it is a consequence of aninfinite-dimensional version of Ths. 12.57 and 12.73 in [20]); the density process is equal to the martingale

$$
\exp \left(\sum_{i=1}^{+\infty} \int_{0}^{1} A_{i}(s) \mathrm{d} B_{i}(s)-\frac{1}{2} \int_{0}^{1}\left|A_{i}(s)\right|^{2} \mathrm{~d} s\right)
$$

This proves the lemma. 
Coming back to the proof of Theorem 2.1, let $\mu \in \mathcal{G}(h, m)$; for all bounded positive measurable function $F$ from $\mathcal{C} \times \mathcal{M}(\mathcal{C})$ to $\mathbb{R}$ we have

$$
\begin{aligned}
& C_{Q^{\mu}}^{!}\left(\exp \left(h(X(0), \Gamma(0))+H^{\Phi}(X, \Gamma)\right) F(X, \Gamma)\right) \\
& =\int_{\mathcal{M}\left(\mathbb{R}^{d}\right) \times \mathcal{M}(\mathcal{C}) \times \mathcal{C}} \mathrm{e}^{h(X(0), \gamma)+H^{\Phi}(X, \Gamma)} F\left(X, \Gamma-\delta_{X}\right) \Gamma(\mathrm{d} X) Q^{\gamma}(\mathrm{d} \Gamma) \mu(\mathrm{d} \gamma) \\
& =\int_{\mathcal{M}\left(\mathbb{R}^{d}\right)} \sum_{i \in \mathbb{N}^{*}} \mathrm{e}^{h\left(\theta_{i}(\gamma), \gamma\right)} \int_{\mathcal{M}(\mathcal{C})} \mathrm{e}^{H^{\Phi}\left(\Theta_{i}(\Gamma), \Gamma\right)} F\left(\Theta_{i}(\Gamma), \Gamma-\delta_{\Theta_{i}(\Gamma)}\right) Q^{\gamma}(\mathrm{d} \Gamma) \mu(\mathrm{d} \gamma)
\end{aligned}
$$

By Lemma 2.6, this is also equal to

$$
\begin{aligned}
\int_{\mathcal{M}\left(\mathbb{R}^{d}\right)} \sum_{i \in \mathbb{N}^{*}} \mathrm{e}^{h\left(\theta_{i}(\gamma), \gamma\right)} \int_{\mathcal{M}(\mathcal{C}) \times \mathcal{C}} F(X, \Gamma) Q^{\gamma-\delta_{\theta_{i}(\gamma)}(\mathrm{d} \Gamma) \otimes \varpi^{\theta_{i}(\gamma)}(\mathrm{d} X) \mu(\mathrm{d} \gamma)} \\
=\mathcal{C}_{\mu}^{!}\left(\mathrm{e}^{h(x, \gamma)} \int_{\mathcal{M}(\mathcal{C})} \int_{\mathcal{C}} F(X, \Gamma) Q^{\gamma}(\mathrm{d} \Gamma) \otimes \varpi^{x}(\mathrm{~d} X)\right) .
\end{aligned}
$$

Using Proposition 1.1, it is also equal to

$$
\int_{\mathcal{M}\left(\mathbb{R}^{d}\right)} \int_{\mathbb{R}^{d}}\left(\int_{\mathcal{M}(\mathcal{C})} \int_{\mathcal{C}} F(X, \Gamma) Q^{\gamma}(\mathrm{d} \Gamma) \otimes \varpi^{x}(\mathrm{~d} X)\right) \mu(\mathrm{d} \gamma) \otimes m(\mathrm{~d} x)=\int_{\mathcal{M}(\mathcal{C})} \int_{\mathcal{C}} F(X, \Gamma) Q^{\mu}(\mathrm{d} \Gamma) \otimes \varpi^{m}(\mathrm{~d} X) .
$$

Therefore, thanks to the last implication of Proposition 1.1, we obtain that $Q^{\mu}$ is a Gibbs field in $\mathcal{G}\left(h \circ \operatorname{pr}_{0}\right.$ $\left.+H, \varpi^{m}\right)$.

When $\mu$ is no more Gibbs but only canonical Gibbs, i.e. $\mu \in \mathcal{G}_{c}(h, m)$, then $\mu$ is a mixture of Gibbs fields in $(\mathcal{G}(h, z m))_{z \in \mathbb{R}^{+}}(6)$. So, $Q^{\mu}$ is a mixture of elements in $\left(\mathcal{G}\left(h \circ \mathrm{pr}_{0}+H, z \varpi^{m}\right)\right)_{z \in \mathbb{R}^{+}}$which means exactly that $Q^{\mu} \in \mathcal{G}_{c}\left(h \circ \mathrm{pr}_{0}+H, \varpi^{m}\right)$.

In the following lemma we prove that the projection at time $t$ of a Gibbs field on $\mathcal{C}$ is a Gibbs field on $\mathbb{R}^{d}$.

Lemma 2.7. Let $m$ be a $\sigma$-finite reference measure on $\mathbb{R}^{d}, H$ a local Hamiltonian on $\mathcal{C}, P$ a Gibbs field in $\mathcal{G}\left(H, \varpi^{m}\right)$ and $t \in[0,1]$. Let us assume that the measure $m_{t}=\varpi^{m} \circ \mathrm{pr}_{t}^{-1}$ is $\sigma$-finite; if we denote by $h_{t}(x, \gamma)$ the following expression:

$$
\begin{aligned}
h_{t}(x, \gamma) & =\log C_{P}^{!}\left(\mathrm{e}^{H(X, \Gamma)} \mid X(t)=x, \Gamma(t)=\gamma\right) \\
& =-\log \int_{\mathcal{C}} \int_{\mathcal{M}(\mathcal{C})} \mathrm{e}^{-H(X, \Gamma)} \varpi^{m}(\mathrm{~d} X \mid X(t)=x) \otimes P(\mathrm{~d} \Gamma \mid \Gamma(t)=\gamma)
\end{aligned}
$$

then the probability measure $P \circ \mathrm{pr}_{t}^{-1}$ is a Gibbs field which belongs to $\mathcal{G}\left(h_{t}, m_{t}\right)$.

Proof. Let $P \in \mathcal{G}\left(H, \varpi^{m}\right)$; using the characterization of Gibbs fields of Proposition 1.1, by projection at time $t \in[0,1]$, we obtain

$$
C_{P}^{!}\left(\mathrm{e}^{H(X, \Gamma)} \mid X(t)=x, \Gamma(t)=\gamma\right) C_{P_{t}}^{!}(d(x, \gamma))=m_{t} \otimes P_{t}(d(x, \gamma))
$$

where $P_{t}:=P \circ \mathrm{pr}_{t}^{-1}$. Thanks to the last implication of Proposition 1.1, this proves that $P_{t}$ is a Gibbs field in $\mathcal{G}\left(h_{t}, m_{t}\right)$. 
Remark. Let $\mu$ be a Gibbs field on $\mathbb{R}^{d}$ with respect to any reference measure $m$. Then, for each $\left.\left.t \in\right] 0,1\right]$, $Q^{\mu} \circ \mathrm{pr}_{t}$ is a Gibbs field on $\mathbb{R}^{d}$ with respect to the reference measure $m_{t}$, which is absolutely continuous with respect to the Lebesgue measure. This is a regularization property for the solution of system (13).

The above lemma has the following consequence for finite volume:

Lemma 2.8. Let $\mu$ be in $\mathcal{P}\left(\mathcal{M}_{\mathcal{E}}\left(\mathbb{R}^{d}\right)\right)$; then, for all $\left.\left.t \in\right] 0,1\right]$ and $\Lambda \in \mathcal{B}\left(\mathbb{R}^{d}\right)$,

$$
Q_{t}^{\mu}\left(d \gamma_{\Lambda} \mid \gamma_{\Lambda^{c}}\right) \ll \pi_{\Lambda}^{\lambda}\left(d \gamma_{\Lambda}\right) \quad Q_{t}^{\mu}-a . s
$$

Proof. It is sufficient to prove this lemma for $\mu=\delta_{\gamma^{\prime}}$, since the absolute continuity property remains true by randomizing the initial condition. Let $\left.\left.\gamma^{\prime} \in \mathcal{M}_{\mathcal{E}}\left(\mathbb{R}^{d}\right), t \in\right] 0,1\right], \Lambda \in \mathcal{B}\left(\mathbb{R}^{d}\right)$ and $A \subset \mathcal{M}(\Lambda)$ such that $\pi_{\Lambda}^{\lambda}(A)=0$. For $Q_{t}^{\gamma^{\prime}}$-almost every $\gamma_{\Lambda^{c}}$,

$$
\begin{aligned}
Q_{t}^{\gamma^{\prime}}\left(\gamma_{\Lambda} \in A \mid \gamma_{\Lambda^{c}}\right)=\sum_{n \in \mathbb{N}} & \sum_{\left\{i_{1}, i_{2}, \ldots, i_{n}\right\} \subset \mathbb{N}^{*}} Q^{\gamma^{\prime}}\left(\Gamma(t)(\Lambda)=n, X_{i_{1}}(t), \ldots, X_{i_{n}}(t) \in \Lambda \mid \Gamma(t)_{\Lambda^{c}}=\gamma_{\Lambda^{c}}\right) \\
& \times Q^{\gamma^{\prime}}\left(\delta_{X_{i_{1}}(t)}+\ldots+\delta_{X_{i_{n}}(t)} \in A \mid \Gamma(t)_{\Lambda^{c}}=\gamma_{\Lambda^{c}}, \Gamma(t)(\Lambda)=n, X_{i_{1}}(t), \ldots, X_{i_{n}}(t) \in \Lambda\right) .
\end{aligned}
$$

But by Lemma 2.6,

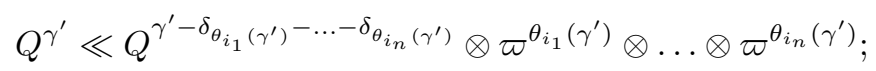

so, the law of $\left(X_{i_{1}}(t), \ldots, X_{i_{n}}(t)\right)$ is absolutely continuous with respect to $\lambda^{\otimes n}$ under $Q^{\gamma^{\prime}}\left(\mid \Gamma(t)_{\Lambda^{c}}=\gamma_{\Lambda^{c}}\right.$, $\left.\Gamma(t)(\Lambda)=n, X_{i_{1}}(t), \ldots, X_{i_{n}}(t) \in \Lambda\right)$ which implies that

$$
Q_{t}^{\gamma^{\prime}}\left(\gamma_{\Lambda} \in A \mid \gamma_{\Lambda^{c}}\right)=0
$$

\subsection{Gibbs fields on $\mathcal{C}$ as laws of interacting Brownian particles}

In the following theorem we prove that Gibbs fields associated to the local Hamiltonian $h \circ \mathrm{pr}_{0}+H^{\Phi}$ are weak solutions of system (13) with initial law a $h$-Gibbs field.

Theorem 2.2. Let $m$ be a $\sigma$-finite reference measure on $\mathbb{R}^{d}$ and $P$ a tempered canonical Gibbs field in $\mathcal{G}_{c}\left(h \circ \mathrm{pr}_{0}+H^{\Phi}, \varpi^{m}\right)$, where $h$ is any local Hamiltonian on $\mathbb{R}^{d}$; if there exists $\left.\eta \in\right] 0,1[$ such that the random variable

$$
\zeta=\sup _{X \in \Gamma} \sup _{0 \leq t \leq 1} \frac{|X(t)-X(0)|}{(1+|X(0)|)^{\eta}}
$$

has a moment of order $\frac{2 d}{1-\eta}$ under $P$, then $P$ is equal to the law $Q^{\mu}$ of the weak solution of (15), with an initial distribution $\mu=P_{0}$ being a canonical Gibbs field in $\mathcal{G}_{c}(h, m)$. Moreover, if $P \in \mathcal{G}\left(H^{\Phi}(X, \Gamma)+h(X(0), \Gamma(0)), \varpi^{m}\right)$, then $P_{0} \in \mathcal{G}(h, m)$.

The following lemma explains why we choose a moment assumption on $\zeta$ in the Theorem 2.2.

Lemma 2.9. Let $\psi$ be a multibody superstable and lower regular interaction, and $\mu \in \mathcal{G}_{c}\left(h^{\psi}, m\right)$ be a Gibbs field on $\mathbb{R}^{d}$ for the local Hamiltonian $h^{\psi}$; then, for every $\left.\eta \in\right] 0,1[$, the variable $\zeta$ defined in (30) admits finite moments of any order under $Q^{\mu}$.

Proof. To prove this lemma, let us show an estimate for the tail of the law of $\zeta$ under $Q^{\mu}$. Using Propositions 2.2 and 2.3 with $\varepsilon=\frac{1}{2}$, we obtain

$$
\begin{aligned}
Q^{\mu}(\zeta \geq u) & \leq Q^{\mu}\left(\zeta \geq u, \mathcal{E}(\Gamma(0)) \leq u^{\frac{1}{5}}\right)+Q^{\mu}\left(\zeta \geq u, \mathcal{E}(\Gamma(0)) \geq u^{\frac{1}{5}}\right) \\
& \leq a_{1} u^{\frac{2}{5}} \exp \left(-b_{1} u^{\frac{1}{10}}\right)+a_{4} \exp \left(-b_{4} u^{\frac{1}{5}}\right)
\end{aligned}
$$


Proof of Theorem 2.2. Let $P$ be a tempered canonical Gibbs field; we write $P^{\gamma}=P(. \mid \Gamma(0)=\gamma)$ and $\tilde{P}^{\gamma}=$ $P^{\gamma} \circ \Theta^{-1}$, where $\Theta$ is defined in (3). Since we would like to use known results about the lattice case, we are now studying the probability measure $\tilde{P}^{\gamma}$ on $\mathcal{C}^{\mathbb{N}^{*}}$.

Lemma 2.10. For $P_{0}$-almost all $\gamma, \tilde{P}^{\gamma}$ is a Gibbs measure on $\mathcal{C}^{\mathbb{N}^{*}}$ associated to the Hamiltonian $\tilde{H}$ defined, for every $i \in \mathbb{N}^{*}$, by

$$
\begin{aligned}
\tilde{H}_{\{i\}}(w)= & \frac{1}{2} \sum_{j \neq i}\left(\varphi\left(w_{i}(1)-w_{j}(1)\right)-\varphi\left(w_{i}(0)-w_{j}(0)\right)\right. \\
& \left.-\int_{0}^{1}\left(\Delta \varphi+\frac{1}{2}|\nabla \varphi|^{2}\right)\left(w_{i}(s)-w_{j}(s)\right) \mathrm{d} s\right) \\
& +\sum_{j<k, j \neq i, k \neq i} \frac{1}{4} \int_{0}^{1}\left[\nabla \varphi\left(w_{i}(s)-w_{j}(s)\right) \cdot \nabla \varphi\left(w_{i}(s)-w_{k}(s)\right)\right. \\
& +\nabla \varphi\left(w_{j}(s)-w_{i}(s)\right) \cdot \nabla \varphi\left(w_{j}(s)-w_{k}(s)\right) \\
& \left.+\nabla \varphi\left(w_{k}(s)-w_{i}(s)\right) \cdot \nabla \varphi\left(w_{k}(s)-w_{j}(s)\right)\right] \mathrm{d} s
\end{aligned}
$$

where $w=\left(w_{j}\right)_{j \in \mathbb{N}^{*}}$ is the canonical variable on $\mathcal{C}^{\mathbb{N}^{*}}$ and the reference measure is the infinite product of Wiener measures $\otimes_{i \in \mathbb{N}^{*}} \varpi^{\theta_{i}(\gamma)}$ on $\mathcal{C}^{\mathbb{N}^{*}}$.

Proof. Since the measure $P$ on $\mathcal{M}(\mathcal{C})$ satisfies the equations given in Definition 1.2 , it is easy to see that the so-called DLR equations are satisfied by $P^{\gamma}$ on $\mathcal{C}^{\mathbb{N}^{*}}$.

Now, we would like to use Theorem 4.9 in [3], which proves that every Gibbs measure on $\mathcal{C}^{\mathbb{N}^{*}}$ associated to the Hamiltonian $\left(\tilde{H}_{\{i\}}\right)_{i \in \mathbb{N}^{*}}$ is a gradient diffusion as soon as certain assumptions are fullfilled. Let us verify that these assumptions are indeed satisfied in our situation.

Lemma 2.11. For P-almost all $\gamma, \tilde{P}^{\gamma}$ satisfies

$$
\forall t \in[0,1], \quad \forall i \in \mathbb{N}^{*}, \quad E_{\tilde{P}^{\gamma}}\left(\left|w_{i}(t)\right|\right)<+\infty,
$$

and

$$
E_{\tilde{P}^{\gamma}}\left(\sum_{j \in \mathbb{N}^{*}} \int_{0}^{1}\left|\nabla_{j} \nabla_{i} \tilde{h}_{i}(w(t))\right|^{2} \mathrm{~d} t\right)<+\infty
$$

where

$$
\tilde{h}_{i}\left(\left(x_{j}\right)_{j}\right)=\sum_{j \neq i} \varphi\left(x_{i}-x_{j}\right),\left(x_{j}\right)_{j} \in\left(\mathbb{R}^{d}\right)^{\mathbb{N}^{*}},
$$

and $\nabla_{j} \nabla_{i} \tilde{h}_{i}$ is the second derivative of $\tilde{h}_{i}$ with respect to the variables $x_{i}$ and $x_{j}$. Moreover, the function $\tilde{H}_{\{i\}}$ is $L^{2}\left(\otimes_{i \in \mathbb{N}^{*}} \varpi^{\theta_{i}(\gamma)}\right)$-differentiable and we have

$$
E_{\tilde{P} \gamma}\left(\int_{0}^{1}\left|D_{r}^{i} \tilde{H}_{\{i\}}\right| \mathrm{d} r\right)<+\infty .
$$

Proof. Remark that (32) (respectively (33)) corresponds to the property (2.12) (respectively (4.10)) in [3].

We denote by $\tilde{\zeta}$ the following function from $\mathcal{C}^{\mathbb{N}^{*}}$ to $\mathbb{R}$

$$
\tilde{\zeta}(w)=\sup _{i \in \mathbb{N}^{*}} \sup _{0 \leq t \leq 1} \frac{\left|w_{i}(t)-w_{i}(0)\right|}{\left(1+\left|w_{i}(0)\right|\right)^{\eta}}
$$


For all $i \in \mathbb{N}^{*}$ and all $t \in[0,1]$

$$
\left|w_{i}(t)\right| \leq\left|w_{i}(0)\right|+\tilde{\zeta}(w)\left(1+\left|w_{i}(0)\right|\right)^{\eta}
$$

implies

$$
\begin{aligned}
\sum_{j \in \mathbb{N}^{*}} \int_{0}^{1}\left|\nabla_{j} \nabla_{i} \tilde{h}_{i}(w(t))\right|^{2} \mathrm{~d} t & =\sum_{j \leq \beta(\tilde{\zeta}, \gamma, i)} \int_{0}^{1}\left|\nabla \nabla \varphi\left(w_{i}(t)-w_{j}(t)\right)\right|^{2} \mathrm{~d} t \\
& \leq\|\nabla \nabla \varphi\|_{\infty}^{2} \beta(\tilde{\zeta}, \gamma, i) \\
& \leq\|\nabla \nabla \varphi\|_{\infty}^{2}\left(K_{3}(\gamma, i)+K_{4}(\gamma, i) \tilde{\zeta}^{\frac{d}{1-\eta}}\right)
\end{aligned}
$$

where $K_{3}$ and $K_{4}$ are the functions introduced in Lemma 2.5. Since $\tilde{\zeta}$ admits a moment of order $\frac{2 d}{1-\eta}>1$ under $\tilde{P}^{\gamma}$, equations (32) and (33) are proved.

$\tilde{H}_{\{i\}}$ is obviously $L^{2}\left(\otimes_{i \in \mathbb{N}^{*}} \varpi^{\theta_{i}(\gamma)}\right)$-differentiable, and there exists a constant $C_{1}$ such that

$$
\int_{0}^{1}\left|D_{r}^{i} \tilde{H}_{\{i\}}\right| \mathrm{d} r \leq C_{1} \beta(\tilde{\zeta}, \gamma, i) \leq C_{1}\left(K_{3}(\gamma, i)+K_{4}(\gamma, i) \tilde{\zeta}^{\frac{d}{1-\eta}}\right)^{2} .
$$

Thanks to (32-34), we can apply Theorem 4.9 in [3] to conclude that $P^{\gamma}$ is a weak solution of system (15) with initial condition $\mu=\delta_{\gamma}$. Therefore, $P$ is a weak solution at (15) with initial law $\mu=P_{0}$ which we have to identify.

If $P$ is a Gibbs field on $\mathcal{C}$, then by Lemma $2.7, P_{0}$ is a Gibbs field on $\mathbb{R}^{d}$ with reference measure $m$ and local Hamiltonian $\bar{h}$ given by: $\forall x \in \mathbb{R}^{d}, \gamma \in \mathcal{M}\left(\mathbb{R}^{d}\right)$ such that $\gamma(x)=0$,

$$
\bar{h}(x, \gamma)=\log C_{P}^{!}\left(\exp \left(h \circ \operatorname{pr}_{0}+H^{\Phi}\right) \mid \Gamma(0)=\gamma, X(0)=x\right) .
$$

Let $i \in \mathbb{N}^{*}$ such that $x=\theta_{i}\left(\gamma+\delta_{x}\right)$, then

$$
\begin{aligned}
\bar{h}(x, \gamma) & =\log E_{P}\left(\exp \left(H^{\Phi}\left(\Theta_{i}(\Gamma), \Gamma-\delta_{\Theta_{i}(\Gamma)}\right)+h(x, \gamma)\right) \mid \Gamma(0)=\gamma\right) \\
& =h(x, \gamma)+\log E_{P^{\gamma}}\left(\exp \left(H^{\Phi}\left(\Theta_{i}(\Gamma), \Gamma \backslash \Theta_{i}(\Gamma)\right)\right)\right) .
\end{aligned}
$$

As in the proof of Lemma 2.6, $\exp \left(H^{\Phi}\left(\Theta_{i}(\Gamma), \Gamma \backslash \Theta_{i}(\Gamma)\right)\right.$ is a $Q^{\gamma}$-exponential martingale evaluated at time 1. Since $P^{\gamma}=Q^{\gamma}, \bar{h}(x, \gamma)=h(x, \gamma)$ and $P_{0} \in \mathcal{G}(h, m)$.

Now, if $P$ is not Gibbs but only canonical Gibbs, i.e. a mixture of Gibbs fields, we can easily deduce that $P_{0} \in \mathcal{G}_{c}(h, m)$.

\section{Some Applications And Generalizations}

We now apply our results to the problem of time reversal. We also generalize Theorem 2.2 to general Gibbs fields. To this end, we need some new tools presented in the following Section 3.1.

\subsection{Integration by parts formulae under Campbell measures}

Let $\mathbf{E}$ denote the set of step-functions from $[0,1]$ to $\mathbb{R}^{d}$ and $\mathbf{F}_{b}$ the set of bounded functions from $\mathbb{R}^{d} \times \mathcal{M}\left(\mathbb{R}^{d}\right)$ to $\mathbb{R}$ which vanish for $|x|$ and $\mathcal{E}(\gamma)$ sufficiently large and with bounded derivatives with respect to the variable $x . \mathcal{W}$ denotes the set of following functionals from $\mathcal{C}$ to $\mathbb{R}: f\left(X(0), X\left(t_{1}\right), \ldots, X\left(t_{n}\right)\right)$, where $f$ is a 
continuously differentiable function with compact support. Similarly, $\overline{\mathcal{W}}$ is the set of following functionals from $\mathcal{C} \times \mathcal{M}(\mathcal{C})$ to $\mathbb{R}$ : $\bar{f}\left(X(0), X\left(t_{1}\right), \ldots, X\left(t_{n}\right), \Gamma\right)$ where $\bar{f}$ is a bounded function which vanishes for $|X(0)|,\left|X\left(t_{1}\right)\right|, \ldots,\left|X\left(t_{n}\right)\right|, \mathcal{E}(\Gamma(0))$ sufficiently large and with bounded derivatives with respect to the $n+1$ first variables. $D$ is the Malliavin derivation operator on $\mathcal{C}$ and $D_{g}$ the derivation in the direction $g \in \mathbf{E}$.

We now exhibit in the next Propositions 3.1 and 3.2 an integration by parts formula for canonical Gibbs fields on $\mathbb{R}^{d}$ and $\mathcal{C}$. For the proofs, we refer to [5].

Proposition 3.1. Let $h$ be a local Hamiltonian on $\mathbb{R}^{d}$ which is differentiable with respect the first variable for each $(x, \gamma)$ such that $\mathcal{E}(\gamma)<+\infty$. Let $\mu \in \mathcal{P}\left(\mathcal{M}_{\mathcal{E}}\left(\mathbb{R}^{d}\right)\right)$ satisfying $\mu\left(\mathcal{M}_{h, \lambda, c}\left(\mathbb{R}^{d}\right)\right)=1$ and, such that for every $M>0$,

$$
C_{\mu}^{!}\left(\left(1+\mathrm{e}^{h(x, \gamma)}\right)\left(1+\left|\nabla_{x} h(x, \gamma)\right|\right) \mathbb{1}_{[0, M]^{2}}(|x|, \mathcal{E}(\gamma))\right)<+\infty ;
$$

then $\mu \in \mathcal{G}_{c}(h, \lambda)$ if and only if

$$
\forall f \in \mathbf{F}_{b}, \quad C_{\mu}^{!}\left(\nabla_{x} f\right)=C_{\mu}^{!}\left(f \nabla_{x} h\right) .
$$

Proposition 3.2. Let $H$ be a local Hamiltonian on $\mathcal{C}$ and $P$ a tempered canonical Gibbs field in $\mathcal{G}\left(H, \varpi^{\lambda}\right)$; we suppose $H \quad D$-differentiable with respect the first variable for $P$-almost all $\Gamma$; if the following integrability property holds: $\forall M>0, \forall t \in[0,1]$

$$
C_{P}^{!}\left(\left(|X(t)|+\int_{0}^{1}\left|D_{s} H\right| \mathrm{d} s\right) \mathbb{I}_{[0, M]^{2}}(|X(0)|, \mathcal{E}(\Gamma(0)))\right)<+\infty,
$$

then $P$ satisfies the following equation: $\forall g \in \mathbf{E}, \forall F \in \overline{\mathcal{W}}$,

$$
C_{P}^{!}\left(F(X, \Gamma) \int_{0}^{1} g(s) \mathrm{d} X(s)\right)=C_{P}^{!}\left(D_{g} F(X, \Gamma)-F(X, \Gamma) D_{g} H(X, \Gamma)\right) .
$$

Remark. The duality equation (37) is based on the famous integration by parts formula on $\mathcal{C}$ which characterizes the Wiener measure, $c f$. [15]. Later, it has been generalize in [30] to characterize the Gibbs fields on $\mathcal{C}$. The Proposition 3.2 is another generalization to canonical Gibbs fields on $\mathcal{C}$. Equation (37) is in fact satisfied for a larger class of probability measures on $\mathcal{M}(\mathcal{C})$. For example, in the following proposition we prove that it is satisfied for $H=H^{\Phi}$ and $P=Q^{\mu}$, where $\mu$ is any probability measure on $\mathcal{M}_{\mathcal{E}}\left(\mathbb{R}^{d}\right.$ ) (not necessarily a Gibbs field).

Proposition 3.3. Let $\mu \in \mathcal{P}\left(\mathcal{M}_{\mathcal{E}}\left(\mathbb{R}^{d}\right)\right)$; then for every $g \in \mathbf{E}$ and $F \in \overline{\mathcal{W}}$, the following duality equation holds:

$$
C_{Q^{\mu}}^{!}\left(F(X, \Gamma) \int_{0}^{1} g(s) \mathrm{d} X(s)\right)=C_{Q^{\mu}}^{!}\left(D_{g} F(X, \Gamma)-F(X, \Gamma) D_{g} H^{\Phi}(X, \Gamma)\right) .
$$

Proof. Let $g \in \mathbf{E}$ and $F \in \overline{\mathcal{W}}$ :

$$
\begin{gathered}
g(s)=u_{1} \mathbb{I}_{\left[0, t_{1}[\right.}(s)+u_{2} \mathbb{I}_{\left[t_{1}, t_{2}[\right.}(s)+\ldots u_{n} \mathbb{I}_{\left[t_{n-1}, t_{n}[\right.}(s), \\
F(X, \Gamma)=\tilde{F}\left(X(0), X\left(t_{1}\right), \ldots, X\left(t_{n}\right), \Gamma\right) \mathbb{1}_{[0, M]}(\mathcal{E}(\Gamma(0))) .
\end{gathered}
$$

We note $X_{i}=\Theta_{i}(\Gamma), x_{i}=X_{i}(0)$. First, let us prove that all the terms in (38) are well defined.

Choose $l \in \mathbb{R}$ such that $F(X, \Gamma)=0$ as soon as $|X(0)| \geq l$; then, for all $t \in[0,1]$ and $i \in \mathbb{N}^{*}$ such that $X_{i}(0) \in B(0, l)$, we have $|X(i)(t)| \leq l+\zeta(1+l)^{\frac{1}{2}}$, where $\zeta$ is defined in (30) and $\eta=\frac{1}{2}$. From Lemma 2.1 which 
controls the number of points of $\Gamma$ in $B(0, l)$ we deduce

$$
\begin{aligned}
C_{Q^{\mu}}^{!}\left(\left|F(X, \Gamma) \int_{0}^{1} g(s) \mathrm{d} X(s)\right|\right) \leq & \int_{\mathcal{M}(\mathcal{C})} \int_{\mathcal{C}}\left(\left|u_{1}\right|\left|X\left(t_{1}\right)-X(0)\right|+\cdots+\left|u_{n}\right|\left|X\left(t_{n}\right)-X\left(t_{n-1}\right)\right|\right) \\
& \times\left|\tilde{F}\left(X, \Gamma-\delta_{X}\right)\right| \mathbb{I}_{[0, M]}(\mathcal{E}(\Gamma(0))) \Gamma(\mathrm{d} X) Q^{\mu}(\mathrm{d} \Gamma) \\
\leq & \int_{\mathcal{M}(\mathcal{C})} 2\|\tilde{F}\|_{\infty} \max \left(\left|u_{1}\right|, \ldots,\left|u_{n}\right|\right)\left(l+\zeta(1+l)^{\frac{1}{2}}\right) M l^{d} \mathbb{I}_{[0, M]}(\mathcal{E}(\Gamma(0))) Q^{\mu}(\mathrm{d} \Gamma) .
\end{aligned}
$$

Thanks to Proposition 2.2 the variable $\zeta \mathbb{1}_{[0, M]}(\mathcal{E}(\Gamma(0)))$ has a finite $Q^{\mu}$-moment, so that the left term in equation (38) is well defined. There is no problem for the term $C_{Q^{\mu}}^{!}\left(D_{g}^{X} F(X, \Gamma)\right)$ because $D_{g}^{X} F(X, \Gamma)$ is bounded. About the last term, we remarked in Lemma 2.11, that $D_{g} H^{\Phi}\left(X_{i}, \Gamma\right)$ is well defined and that there exists a constant $C_{1}$ such that

$$
\left|D_{g} H^{\Phi}\left(X_{i}, \Gamma\right)\right| \leq C_{1} \beta(\zeta, \gamma, i)^{2} .
$$

By Lemma 2.5, there exists two constants $C_{2}, C_{3}$ such that

$$
C_{Q^{\mu}}^{!}\left(\left|D_{g} H^{\Phi}(X, \Gamma) F(X, \Gamma)\right|\right) \leq \int_{\mathcal{M}(\mathcal{C})} M L^{d} C_{1}\left(C_{2}+C_{3} \zeta^{4 d}\right)\|F\|_{\infty} \mathbb{I}_{[0, M]}(\mathcal{E}(\Gamma(0))) Q^{\mu}(\mathrm{d} \Gamma)
$$

which is also finite since $\zeta \mathbb{1}_{[0, M]}(\mathcal{E}(\Gamma(0)))$ has a finite moment of order $4 d$ under $Q^{\mu}$. Now, we can do the following computations: due to Lemma 2.6 , we have

$$
\begin{aligned}
C_{Q^{\mu}}^{!}\left(F(X, \Gamma) \int_{0}^{1} g(s) \mathrm{d} X(s)\right)= & \int_{\mathcal{M}\left(\mathbb{R}^{d}\right)} \sum_{i} \int_{\mathcal{M}(\mathcal{C})}\left(F\left(X_{i}, \Gamma-\delta_{X_{i}}\right) \int_{0}^{1} g(s) \mathrm{d} X_{i}(s)\right) Q^{\gamma}(\mathrm{d} \Gamma) \mu(\mathrm{d} \gamma) . \\
= & \int_{\mathcal{M}\left(\mathbb{R}^{d}\right)} \sum_{i} \int_{\mathcal{M}(\mathcal{C})}\left(\exp \left(-H^{\Phi}(X, \Gamma)\right) F(X, \Gamma-\delta X) \int_{0}^{1} g(s) \mathrm{d} X(s)\right) \\
& \times \varpi^{x_{i}} \otimes Q^{\gamma-\delta_{x_{i}}(\mathrm{~d} X, \mathrm{~d} \Gamma) \mu(\mathrm{d} \gamma) .}
\end{aligned}
$$

Using the integration by parts formula under $\varpi^{x_{i}}$ and localizing the $L^{2}$-derivation, the last expression equals

$$
\begin{aligned}
& \int_{\mathcal{M}\left(\mathbb{R}^{d}\right)} \sum_{i} \int_{\mathcal{M}(\mathcal{C})}\left(-D_{g} H^{\Phi}(X, \Gamma) \exp \left(-H^{\Phi}(X, \Gamma)\right) F\left(X, \Gamma-\delta_{X}\right)\right. \\
& \left.\quad+D_{g} F(X, \Gamma-\delta X) \exp \left(-H^{\Phi}(X, \Gamma)\right)\right) \varpi^{x_{i}} \otimes Q^{\gamma-\delta_{x_{i}}}(\mathrm{~d} X, \mathrm{~d} \Gamma) \mu(\mathrm{d} \gamma) \\
& =\int_{\mathcal{M}\left(\mathbb{R}^{d}\right)} \sum_{i} \int_{\mathcal{M}(\mathcal{C})}\left(-D_{g} H^{\Phi}\left(X_{i}, \Gamma\right) F\left(X_{i}, \Gamma-\delta_{X_{i}}\right)+D_{g} F\left(X_{i}, \Gamma-\delta_{X_{i}}\right)\right) Q^{\gamma}(\mathrm{d} \Gamma) \mu(\mathrm{d} \gamma) \\
& =C_{Q^{\mu}}^{!}\left(-D_{g} H^{\Phi}(X, \Gamma) F(X, \Gamma)+D_{g} F(X, \Gamma)\right) .
\end{aligned}
$$

\subsection{Application to reversible measures}

A probability measure $\mu \in \mathcal{P}\left(\mathcal{M}_{\mathcal{E}}\left(\mathbb{R}^{d}\right)\right)$ is called reversible under the dynamics of system (13) if for every $t \in[0,1]$, the processes $(\Gamma(s))_{s \in[0, t]}$ and $(\Gamma(t-s))_{s \in[0, t]}$ have the same law under $Q^{\mu}$.

In [22], Lang proved under strong assumptions that the reversible measures of system (13) are the canonical Gibbs fields in $\mathcal{G}_{c}\left(h^{\varphi}, \lambda\right)$, where $h^{\varphi}$ is defined in (12). We give here another proof of this result under much weaker assumptions on the reversible measures. More precisely, Lang considered only tempered reversible measures which are locally absolutely continuous with respect to the Poisson process with regular integrable densities. In our context, we consider reversible measures which are only tempered a priori. 
Proposition 3.4. A probability measure $\mu \in \mathcal{P}\left(\mathcal{M}_{\mathcal{E}}\left(\mathbb{R}^{d}\right)\right)$ is reversible for the dynamical system (13) if and only if $\mu \in \mathcal{G}_{c}\left(h^{\varphi}, \lambda\right)$.

Proof. First, we introduce the following notations: for $X \in \mathcal{C}, \Gamma \in \mathcal{M}(\mathcal{C})$ and $P \in \mathcal{P}(\mathcal{M}(\mathcal{C}))$ we denote by $\hat{X}$, respectively $\hat{\Gamma}$, the process $(\hat{X}(t))_{t \in[0,1]} \equiv(X(1-t))_{t \in[0,1]}$, respectively $\hat{\Gamma}()=.\Gamma(1-$.$) , and by \hat{P}$ the law of $\hat{\Gamma}$ under $P$.

We now prove that $\mu$ is reversible if $\mu \in \mathcal{G}_{c}\left(h^{\varphi}, \lambda\right)$.

Let $\mu \in \mathcal{G}_{c}\left(h^{\varphi}, \lambda\right)$. By Proposition 2.3, $\mu$ is tempered. Therefore, using Theorem 2.1, $Q^{\mu} \in \mathcal{G}_{c}\left(h^{\varphi} \circ \mathrm{pr}_{0}\right.$ $\left.+H^{\Phi}, \varpi^{\lambda}\right)$. The local Hamiltonian $h^{\varphi} \circ \mathrm{pr}_{0}+H^{\Phi}$ is invariant for time reversal, so $\hat{Q^{\mu}}$ is a canonical Gibbs field in $\mathcal{G}_{c}\left(h^{\varphi} \circ \mathrm{pr}_{0}+H^{\Phi}, \varpi^{\lambda}\right)$ too. Thanks to Theorem 2.2, $\hat{Q}^{\mu}$ is a weak solution of system (15) with initial law $Q^{\mu} \circ \mathrm{pr}_{1}^{-1}$. By uniqueness of the solutions of (15), it is then sufficient to prove that $Q^{\mu} \circ \mathrm{pr}_{1}^{-1}=\mu$ to conclude that $Q^{\mu}=\hat{Q}^{\mu}$. Let us denote by $\nu$ the following probability measure:

$$
\nu=Q^{\mu} \circ \operatorname{pr}_{\frac{1}{2}}^{-1}=\hat{Q}^{\mu} \circ \operatorname{pr}_{\frac{1}{2}}^{-1}
$$

$Q^{\mu}$ and $\hat{Q}^{\mu}$ are Markovian solutions of (15), so $Q^{\mu} \circ \mathrm{pr}_{1}^{-1}$ is the law of $\Gamma\left(\frac{1}{2}\right)$ under $Q^{\nu}$ and, similarly, $\mu=\hat{Q}^{\mu} \circ \mathrm{pr}_{1}^{-1}$ is the law of $\Gamma\left(\frac{1}{2}\right)$ under $Q^{\nu}$. Therefore, $\mu=Q^{\mu} \circ \mathrm{pr}_{1}^{-1}$ and $Q^{\mu}=\hat{Q}^{\mu}$. Applying the same argument on each interval $[0, t]$ instead of $[0,1]$, we prove that $\mu$ is reversible.

We now prove that any tempered reversible measure $\mu$ is a canonical Gibbs field. Let $\vec{u}$ be a vector in $\mathbb{R}^{d}$ and $f$ a function in $\mathbf{F}_{b}$. Writing equality (38) for $g(s)=-\vec{u}$ and $F(X, \Gamma)=f(X(0), \Gamma(0))$, we obtain

$$
C_{Q^{\mu}}^{!}(\vec{u} .(X(0)-X(1)) f(X(0), \Gamma(0)))=C_{Q^{\mu}}^{!}\left(D_{\vec{u}} H^{\Phi}(X, \Gamma) f(X(0), \Gamma(0))\right) .
$$

Similarly, let us write $(38)$ for $g(s)=\vec{u}$ and $F(X, \Gamma)=k(X(0)) f(X(1), \Gamma(1)) \mathbb{1}_{[0, M]}(\mathcal{E}(\Gamma(0)))$, where $k$ is regular bounded function from $\mathbb{R}^{d}$ to $\mathbb{R}$ with compact support:

$$
\begin{array}{r}
C_{Q^{\mu}}^{!}\left(\vec{u} .(X(1)-X(0)) f(X(1), \Gamma(1)) k(X(0)) \mathbb{I}_{[0, M]}(\mathcal{E}(\Gamma(0)))\right) \\
=C_{Q^{\mu}}^{!}\left(-D_{\vec{u}} H^{\Phi}(X, \Gamma) f(X(1), \Gamma(1)) k(X(0)) \mathbb{1}_{[0, M]}(\mathcal{E}(\Gamma(0)))\right. \\
\left.+\vec{u} . \nabla_{x} f(X(1), \Gamma(1)) k(X(0)) \mathbb{I}_{[0, M]}(\mathcal{E}(\Gamma(0)))\right) . \quad(40)
\end{array}
$$

Now, by time reversal, letting $K$ (respectively $M$ ) converge to 1 (respectively to $+\infty$ ), we obtain a new equality which, together with (39), gives

$$
C_{Q^{\mu}}^{!}\left(\left(D_{\vec{u}} H^{\Phi}(X, \Gamma)+D_{\vec{u}}^{X} H(\hat{X}, \hat{\Gamma})\right) f(X(0), \Gamma(0))\right)=C_{Q^{\mu}}^{!}\left(\vec{u} . \nabla_{x} f(X(0), \Gamma(0))\right) .
$$

Let $i_{0}$ be a fixed positive integer; using the expression $(27)$ of $H^{\Phi}\left(X_{i_{0}}, \Gamma\right)$, we compute the term $D_{\vec{u}} H^{\Phi}\left(X_{i_{0}}, \Gamma\right)$ under $Q^{\gamma}$ :

$$
\begin{aligned}
D_{\vec{u}} H^{\Phi}\left(X_{i_{0}}, \Gamma\right)= & \frac{1}{2} \sum_{i \neq i_{0}}\left(\vec{u} . \nabla \varphi\left(X_{i_{0}}(1)-X_{i}(1)\right)-\int_{0}^{1} s \vec{u} . \nabla \Delta \varphi\left(X_{i_{0}}(s)-X_{i}(s)\right) \mathrm{d} s\right) \\
& -\frac{1}{4} \sum_{i \neq i_{0}} \int_{0}^{1} s\left(\vec{u} . \nabla \nabla \varphi\left(X_{i_{0}}(s)-X_{i}(s)\right)\right) \cdot \sum_{j \neq i} \nabla \varphi\left(X_{i}(s)-X_{j}(s)\right) \mathrm{d} s \\
& +\frac{1}{4} \int_{0}^{1} s\left(\sum_{i \neq i_{0}} \nabla \varphi\left(X_{i_{o}}(s)-X_{i}(s)\right)\right) \cdot\left(\sum_{i \neq i_{0}} \vec{u} . \nabla \nabla \varphi\left(X_{i_{o}}(s)-X_{i}(s)\right)\right) \mathrm{d} s .
\end{aligned}
$$


This implies that:

$$
\begin{aligned}
D_{\vec{u}} H^{\Phi}\left(X_{i_{0}}, \Gamma\right)+D_{\vec{u}} H^{\Phi}\left(\hat{X}_{i_{0}}, \hat{\Gamma}\right)= & \frac{1}{2} \sum_{i \neq i_{0}}\left(\vec{u} . \nabla \varphi\left(X_{i_{0}}(1)-X_{i}(1)\right)+\vec{u} . \nabla \varphi\left(X_{i_{0}}(0)-X_{i}(0)\right)\right) \\
& \left.-\frac{1}{2} \sum_{i \neq i_{0}} \int_{0}^{1} \vec{u} \cdot \nabla \Delta \varphi\left(X_{i_{0}}(s)-X_{i}(s)\right) \mathrm{d} s\right) \\
& -\frac{1}{4} \sum_{i \neq i_{0}} \int_{0}^{1}\left(\vec{u} \cdot \nabla \nabla \varphi\left(X_{i_{0}}(s)-X_{i}(s)\right)\right) \cdot \sum_{j \neq i} \nabla \varphi\left(X_{i}(s)-X_{j}(s)\right) \mathrm{d} s \\
& +\frac{1}{4} \int_{0}^{1}\left(\sum_{i \neq i_{0}} \nabla \varphi\left(X_{i_{o}}(s)-X_{i}(s)\right)\right) \cdot\left(\sum_{i \neq i_{0}} \vec{u} . \nabla \nabla \varphi\left(X_{i_{o}}(s)-X_{i}(s)\right)\right) \mathrm{d} s .(4)
\end{aligned}
$$

But, for all $i \in \mathbb{N}^{*}$, the process $X_{i}(t)-X_{i}(0)+\frac{1}{2} \int_{0}^{t} \sum_{j \neq i} \nabla \varphi\left(X_{i}(s)-X_{j}(s)\right) \mathrm{d} s$ is a $Q^{\gamma}$-Brownian motion; so, by Ito formula, we obtain

$$
\begin{aligned}
& E_{Q^{\gamma}}\left(f\left(X_{i_{0}}(0), \Gamma(0)\right)\left(D_{\vec{u}} H^{\Phi}\left(X_{i_{0}}, \Gamma\right)+D_{\vec{u}} H^{\Phi}\left(\hat{X}_{i_{0}}, \hat{\Gamma}\right)\right)\right) \\
& =E_{Q^{\gamma}}\left(f ( X _ { i _ { 0 } } ( 0 ) , \Gamma ( 0 ) ) \left(\vec{u} \cdot \sum_{i \neq i_{0}} \nabla \varphi\left(X_{i_{0}}(0)-X_{i}(0)\right)\right.\right. \\
& -\frac{1}{2} \sum_{i \neq i_{0}} \int_{0}^{1} \vec{u} . \nabla \nabla \varphi\left(X_{i_{0}}(s)-X_{i}(s)\right)\left(\mathrm{d} X_{i}(s)+\frac{1}{2} \sum_{j \neq i} \nabla \varphi\left(X_{i}(s)-X_{j}(s)\right) \mathrm{d} s\right) \\
& \left.\left.+\frac{1}{2} \sum_{i \neq i_{0}} \int_{0}^{1} \vec{u} . \nabla \nabla \varphi\left(X_{i_{0}}(s)-X_{i}(s)\right)\left(\mathrm{d} X_{i_{0}}(s)+\frac{1}{2} \sum_{j \neq i_{0}} \nabla \varphi\left(X_{i_{o}}(s)-X_{j}(s)\right) \mathrm{d} s\right)\right)\right) \\
& =E_{Q^{\gamma}}\left(\left(\vec{u} . \nabla_{x} h^{\varphi}\left(X_{i_{0}}(0), \Gamma(0)\right)\right) f\left(X_{i_{0}}(0), \Gamma(0)\right)\right) .
\end{aligned}
$$

This computation, together with (41), implies that

$$
C_{Q^{\mu}}^{!}\left(\vec{u} . \nabla_{x} h^{\varphi}(X(0), \Gamma(0)) f(X(0), \Gamma(0))\right)=C_{Q^{\mu}}^{!}\left(\vec{u} . \nabla_{x} f(X(0), \Gamma(0))\right) .
$$

Using Proposition 3.1, we conclude that $\mu$ is a canonical Gibbs field associated to the local Hamiltonian $h^{\varphi}$.

Remark. To prove that a reversible measure is a canonical Gibbs fields, we use only $Q^{\mu}=\hat{Q}^{\mu}$ which is weaker than the reversibility.

\subsection{General Gibbs fields on $\mathcal{C}$ as Brownian diffusions; application to time reversal in the non stationary case}

Theorem 3.1 is a generalization of Theorem 2.2 when the local Hamiltonian $H$ is general. First, we prove a lemma which we will use later to identify diffusions. 
Lemma 3.1. Let $\left(b_{t}\right)_{t \in[0,1]}$ a family of $\mathcal{F}_{t}$-adapted functionals from $\mathcal{C} \otimes \mathcal{M}(\mathcal{C})$ to $\mathbb{R}$ and $P$ a probability measure on $\mathcal{M}(\mathcal{C})$ such that $C_{P}^{!}$is $\sigma$-finite; then the following assertions are equivalent:

i) the process

$$
W_{i, t}=: \Theta_{i}(\Gamma)(t)-\Theta_{i}(\Gamma)(0)-\int_{0}^{t} b_{s}\left(\Theta_{i}(\Gamma), \Gamma-\delta_{\Theta_{i}(\Gamma)}\right) \mathrm{d} s, \quad \text { for } i \in \mathbb{N}^{*}, t \in[0,1]
$$

is an infinite family of $\left(P, \mathcal{F}_{t}\right)$-Brownian motions starting from 0 ;

ii) the process

$$
W_{t}:=X(t)-X(0)-\int_{0}^{t} b_{s}(X, \Gamma) \mathrm{d} s, \quad \text { for } t \in[0,1]
$$

is, for all $M>0$, a $\left(\mathbb{I}_{[0, M]}(X(0)) C_{P}^{!}, \mathcal{F}_{t}\right)$-Brownian motion starting from 0.

Proof. Let us first prove that $i$ ) implies ii). For every $s \in[0,1]$, every $\mathcal{F}_{s}$-measurable functional $F_{s}$ in $\overline{\mathcal{W}}$, we have for $t \geq s$

$$
C_{P}^{!}\left(F_{s}(X, \Gamma)\left(W_{t}-W_{s}\right)\right)=\int_{\mathcal{M}\left(\mathbb{R}^{d}\right)} \sum_{i \in \mathbb{N}^{*}} \int_{\mathcal{M}(\mathcal{C})} F_{s}\left(\Theta_{i}(\Gamma), \Gamma-\delta_{\Theta_{i}(\Gamma)}\right)\left(W_{i, t}-W_{i, s}\right) P^{\gamma}(\mathrm{d} \Gamma) P_{0}(\mathrm{~d} \gamma)
$$

Since for all $i \in \mathbb{N}^{*}, W_{i}$ is a $\left(P, \mathcal{F}_{t}\right)$-Brownian motion under $P, W_{i}$ is independent from the $\sigma$-algebra $\mathcal{F}_{0}$ and it is a $\left(P^{\gamma}, \mathcal{F}_{t}\right.$-Brownian motion too $)$. Therefore,

$$
C_{P}^{!}\left(F_{s}(X, \Gamma)\left(W_{t}-W_{s}\right)\right)=0
$$

and $W_{t}$ is an $\mathcal{F}_{t}$-martingale under $\mathbb{I}_{[0, M]}(X(0)) C_{P}^{!}$. Similarly, we can prove that $W_{t}^{2}-t$ is an $\mathcal{F}_{t}$-martingale under $\mathbb{I}_{[0, M]}(X(0)) C_{P}^{!}$which implies $\left.i i\right)$.

Now let us prove that $i$ i) implies $i$ ): let $\gamma \in \mathcal{M}\left(\mathbb{R}^{d}\right)$ and $i \in \mathbb{N}^{*} . W$ is an $\mathcal{F}_{t}$-Brownian motion under $\mathbb{I}_{[0, M]}(X(0)) C_{P}^{!}$and independent from $\mathcal{F}_{0}$. Thus, $W$ is also an $\mathcal{F}_{t}$-Brownian motion under $C_{P}^{!}(\mid X(0)=$ $\left.\theta_{i}(\gamma), \Gamma(0)=\gamma-\delta_{\theta_{i}(\gamma)}\right)$. By the identification

$$
C_{P}^{!}\left((\mathrm{d} X, \mathrm{~d} \Gamma) \mid X(0)=\theta_{i}(\gamma), \Gamma(0)=\gamma-\delta_{\theta_{i}(\gamma)}\right)=P^{\gamma}\left(\left(d \Theta_{i}(\Gamma), d\left(\Gamma-\delta_{\Theta_{i}(\Gamma)}\right)\right)\right)
$$

we deduce that $W_{i}$ is an $\mathcal{F}_{t}$-Brownian motion under $P^{\gamma}$ and also under $P$.

Remark. In the proposition $i$ ) the family of Brownian motions is not supposed to be independent.

Theorem 3.1. Let $P$ be a canonical Gibbs field on $\mathcal{C}$ in $\mathcal{G}_{c}\left(H, \varpi^{m}\right)$, where $m$ is $\sigma$-finite measure on $\mathbb{R}^{d}$ and $H$ a local Hamiltonian which is $L^{2}$-differentiable with respect to the first variable and which satisfies the integrability condition (36); then, denoting by $X_{i}\left(i \in \mathbb{N}^{*}\right)$ the random variable $\Theta_{i}(\Gamma)$, the process

$$
\left(X_{i}(t)-X_{i}(0)-\int_{0}^{t} b_{s}\left(X_{i}, \sum_{j \neq i} \delta_{X_{j}}\right) \mathrm{d} s\right)_{i \in \mathbb{N}^{*}, t \in[0,1]}
$$

is a family of independent $P$-Brownian motions in $\mathbb{R}^{d}$, where $\left(b_{t}(X, \Gamma)\right)_{t \in[0,1]}$ is the adapted process from $\mathcal{C} \times$ $\mathcal{M}(\mathcal{C})$ to $\mathbb{R}$ defined for $\lambda$-almost all $t$ by

$$
b_{t}(X, \Gamma)=-C_{P}^{!}\left(D_{t} H(X, \Gamma) \mid \mathcal{F}_{t}\right) .
$$


Proof. $P$ is a canonical Gibbs field on $\mathcal{C}$; therefore Proposition 1.1 implies that there exists a measure $\tilde{P} \in$ $\mathcal{P}(\mathcal{M}(\mathcal{C}))$ such that $C_{P}^{!}$is absolutely continuous with respect to $\varpi^{m} \otimes \tilde{P}$. Consequently, the finite measure $\mathbb{I}_{[0, M]}(X(0)) C_{P}^{!}$is absolutely continuous with respect to $\varpi^{m} \otimes \tilde{P}$ too; this implies the existence of a process $\left(b_{t}\right)_{t \in[0,1]}$ on $\mathcal{C} \otimes \mathcal{M}(\mathcal{C})$ such that

$$
X(t)-X(0)-\int_{0}^{t} b_{s}(X, \Gamma) \mathrm{d} s
$$

is a $\mathcal{F}_{t}$ Brownian motion starting from 0 under $\mathbb{1}_{[0, M]}(|X(0)|) C_{P}^{!}$, for every $M \geq 0$. From Lemma 3.1 , we deduce that, for each $i \in \mathbb{N}^{*}$,

$$
B_{i}(.)=: X_{i}(.)-X_{i}(0)-\int_{0} b_{s}\left(X_{i}, \sum_{j \neq i} \delta_{X_{j}}\right) \mathrm{d} s
$$

is an $\mathcal{F}_{t}$-Brownian motion with values in $\mathbb{R}^{d}$ under $P$. We now prove that these Brownian motions are independent, when $i$ varies. Using the same argument as in Lemma 2.10, we know that, for all finite subset $\Lambda$ in $\mathbb{N}^{*}, P^{\gamma} \circ \Theta^{-1}$ is absolutely continuous with respect to $\left(\bigotimes_{i \in \Lambda} \varpi^{\theta_{i}(\gamma)}\right) \otimes\left(P^{\gamma} \circ \Theta^{-1}\right)_{\Lambda^{c}}$ on $\mathcal{C}^{\mathbb{N}^{*}}$. So there exists a process $\left(b_{i, t}^{\Lambda}\right)_{i \in \Lambda}$ such that

$$
\left(w_{i}(.)-w_{i}(0)-\int_{0}^{\cdot} b_{i, s}^{\Lambda}(w) \mathrm{d} s\right)_{i \in \Lambda}
$$

is a family of independent $P^{\gamma} \circ \Theta^{-1}$-Brownian motions, where $w=\left(w_{j}\right)_{j \in \mathbb{N}^{*}}$ is the canonical variable in $C^{\mathbb{N}^{*}}$. Using the uniqueness of the semi-martingale decomposition of $w$, we deduce that $\left(B_{i}\right)_{i \in \Lambda}$ is a family of independent Brownian motions under $P^{\gamma}$.

Next we identify the process $\left(b_{t}(X, \Gamma)\right)_{t \in[0,1]}$. Since $X(t)-X(0)-\int_{0}^{t} b_{s}(X, \Gamma) \mathrm{d} s$ is a Brownian motion, it is an $\mathcal{F}_{t}$-martingale under $\mathbb{I}_{[0, K]}(|X(0)|) C_{Q}^{!}$; therefore the following equality holds:

$$
C_{Q}^{!}\left(F_{s}(X, \Gamma)\left(X(t)-X(s)-\int_{s}^{t} b_{r}(X, \Gamma) \mathrm{d} r\right)\right)=0
$$

for every $0 \leq s \leq t \leq 1$, and every $\mathcal{F}_{s}$-measurable functional $F_{s}$ in $\overline{\mathcal{W}}$. This equation together with the integration by parts formula $(37)$ applied to $g=\mathbb{I}_{] s, t]}$ and $F_{s}$, implies that

$$
C_{P}^{!}\left(F_{s}(X, \Gamma)\left(\int_{s}^{t} b_{r}(X, \Gamma)+D_{r} H(X, \Gamma)\right)\right)=0
$$

By classical approximation methods, we deduce easily that, for $s \in \mathbb{Q} \cap[0,1]$ and for $\lambda$-almost every $r \in[0,1]$

$$
C_{P}^{!}\left(\mathbb{I}_{s \leq r} F_{s}(X, \Gamma)\left(b_{r}(X, \Gamma)+D_{r} H(X, \Gamma)\right)\right)=0 .
$$

Letting $s$ converge to $r$, this implies that, for $\lambda$-almost every $r \in[0,1]$,

$$
b_{r}(X, \Gamma)=-C_{Q}^{!}\left(D_{r} H(X, \Gamma) \mid \mathcal{F}_{r}\right)
$$

In Theorem 3.2 which follows, we give an application to the time reversal in the non stationary case.

For simplicity, we denote by $\hat{H}(X, \Gamma)$ the local Hamiltonian $H(\hat{X}, \hat{\Gamma})$.

Theorem 3.2. Let $P$ be a Gibbs field on $\mathcal{C}$ in $\mathcal{G}\left(H, \varpi^{\lambda}\right)$, where $H$ is a local Hamiltonian such that $H$ and $\hat{H}$ are $L^{2}$-differentiable with respect to the first variable and that they satisfy the following integrability condition: $\forall M>0, \forall t \in[0,1]$

$$
C_{P}^{!}\left(\left(|X(t)|+\int_{0}^{1}\left(\left|D_{s} H\right|^{2}+\left|D_{s} \hat{H}\right|^{2}\right) \mathrm{d} s\right) \mathbb{I}_{[0, M]^{2}}(|X(0)|, \mathcal{E}(\Gamma(0)))\right)<+\infty ;
$$


then, if for every $i \in \mathbb{N}^{*}$ we denote by $X_{i}$ the variable $\Theta_{i}(\Gamma)$, there exist two adapted processes $\left(b_{s}\right)_{s \in[0,1]}$ and $\left(\hat{b}_{s}\right)_{s \in[0,1]}$ from $\mathcal{C} \times \mathcal{M}(\mathcal{C})$ to $\mathbb{R}$ such that

$$
\left(X_{i}(t)-X_{i}(0)-\int_{0}^{t} b_{s}\left(X_{i}, \sum_{j \neq i} \delta_{X_{j}}\right) \mathrm{d} s\right)_{i \in \mathbb{N}^{*}, t \in[0,1]}
$$

is a family of independent P-Brownian motions and

$$
\left(X_{i}(t)-X_{i}(0)-\int_{0}^{t} \hat{b}_{s}\left(X_{i}, \sum_{j \neq i} \delta_{X_{j}}\right) \mathrm{d} s\right)_{i \in \mathbb{N}^{*} t \in[0,1]}
$$

is a family of independent $\hat{P}$-Brownian motions too.

The processes $b$ and $\hat{b}$ are called forward and backward drifts associated to $P$; if we denote by $h_{t}$ the local Hamiltonian associated to the Gibbs field $P_{t}=P \circ \mathrm{pr}_{t}^{-1}$, we have for $\lambda$-a.s. every $t \in[0,1]$, and $P_{t}$-almost every $\gamma$,

$$
-\tilde{\nabla}_{x} h_{t}(x, \gamma)=C_{P}^{!}\left(b_{t}(X, \Gamma)+\hat{b}_{1-t}(\hat{X}, \hat{\Gamma}) \mid X(t)=x, \Gamma(t)=\gamma\right) \lambda-a . s .
$$

where $\tilde{\nabla}$ is the weak gradient operator in $H^{1,2}\left(\mathbb{R}^{d}, \lambda\right)$. In the case $d=1$, the equation (49) remains true by substituting the weak gradient operator $\tilde{\nabla}$ by the ordinary gradient operator $\nabla$.

Proof. Since $P$ is a Gibbs field in $\mathcal{G}\left(H, \varpi^{\lambda}\right)$ and $\hat{\varpi}^{\lambda}=\varpi^{\lambda}$ it is clear that $\hat{P} \in \mathcal{G}\left(\hat{H}, \varpi^{\lambda}\right)$. By Theorem $3.1, b_{t}$ and $\hat{b}_{t}$ exist and satisfy (47) and (48).

On the other hand, Lemma 2.7 proves that $P_{t}$ is a Gibbs field in $\mathcal{G}\left(h_{t}, \lambda\right)$, where $h_{t}$ is explicit.

Equality (37) applied to $g=\mathbb{I}_{[s, t]}$ and $F(X, \Gamma)=f(X(t), \Gamma(t))$ implies

$$
\begin{aligned}
C_{\hat{P}}^{!}(f(X(1-t), \Gamma(1-t))(X(1-t)-X(1-s))) & \\
& =(t-s) C_{P}^{!}\left(\nabla_{x} f(X(t), \Gamma(t))-C_{P}^{!}(f(X(t), \Gamma(t))) D_{\mathbb{I}_{[s, t]}} H(X, \Gamma)\right) .
\end{aligned}
$$

Let us divide both sides by $t-s$ and let $t$ converge to $s$; in this way, we obtain for $\lambda$-almost every $t \in[0,1]$,

$$
-C_{\hat{P}}^{!}\left(f(X(1-t), \Gamma(1-t)) \hat{b}_{1-t}(X, \Gamma)\right)=C_{P}^{!}\left(\nabla_{x} f(X(t), \Gamma(t))\right)+C_{P}^{!}\left(f(X(t), \Gamma(t)) b_{t}(X, \Gamma)\right)
$$

which can be written as

$$
-C_{P_{t}}^{!}\left(\nabla_{x} f(x, \gamma)\right)=C_{P_{t}}^{!}\left(f(x, \gamma) q_{t}(x, \gamma)\right)
$$

where $q_{t}(x, \gamma)=C_{P}^{!}\left(\hat{b}_{1-t}(\hat{X}, \hat{\Gamma})+b_{t}(X, \Gamma) \mid X(t)=x, \Gamma(t)=\gamma\right)$. By Lemma $9, C_{P_{t}}^{!}=\mathrm{e}^{-h_{t}} \lambda \otimes P_{t}$; therefore, equation (50) becomes for $P_{t}$-almost every $\gamma$

$$
\int_{\mathbb{R}^{d}} \nabla_{x} f(x, \gamma) \mathrm{e}^{-h_{t}(x, \gamma)} \lambda(\mathrm{d} x)=-\int_{\mathbb{R}^{d}} f(x, \gamma) q_{t}(x, \gamma) \mathrm{e}^{-h_{t}(x, \gamma)} \lambda(\mathrm{d} x) .
$$

Thanks to (46), the function $q_{t}(., \gamma)$ is locally, for $P_{t}$-almost every $\gamma$, in $L^{2}\left(\mathrm{e}^{-h_{t}(x, \gamma)} \lambda(\mathrm{d} x)\right)$ and is equal to the logarithmic derivative of the measure $\mathrm{e}^{-h_{t}(x, \gamma)} \lambda(\mathrm{d} x)(c f .[2])$. Therefore, $-h_{t}(x, \gamma)$ is for $P_{t}$-almost every $\gamma$ in $H^{1,2}\left(\mathbb{R}^{d}, \lambda\right)$ with derivative $q_{t}(x, \gamma)$. 
If $d=1$, equation (51) implies that, for $P_{t}$-almost every $\gamma,-h_{t}(x, \gamma)$ is differentiable for $\lambda$-almost every $x$ and admit $q(x, \gamma)$ as gradient function.

Example. If $b$ is a Markovian drift, then $\hat{b}$ is Markovian too:

$$
b_{t}(X, \Gamma)=b_{t}(X(t), \Gamma(t)), \quad \hat{b}_{t}(X, \Gamma)=\hat{b}_{t}(X(t), \Gamma(t))
$$

and formula (49) becomes

$$
-\tilde{\nabla}_{x} h_{t}(x, \gamma)=b_{t}(x, \gamma)+\hat{b}_{1-t}(x, \gamma) .
$$

In particular the assumptions of Theorem 3.2 are satisfied for the Gibbs field $Q^{\mu}$ in $\mathcal{G}\left(h \circ \mathrm{pr}_{0}+H^{\Phi}, \varpi^{\lambda}\right)$ solutions of system (15) with $\mu$ a tempered Gibbs field associated to the local Hamiltonian $h$.

\subsection{The case $d \geq 4$}

Some results of this paper remain true in the case $d \geq 4$.

If $d=4$, Theorems 2.1 and 2.2 are still true ( $c f$. [6] for the detailed proofs). But, we do not know how to prove Lemma 2.9. So the moment assumption on $\zeta$ in Theorem 2.2 remains unjustified. Similarly, the proof of Proposition 3.4 is no more valid, because it uses moments of the function $\zeta$.

If $d>4$, there does not exist any proof for the existence of weak solution of system (15) except in the stationary case dealt by Lang. In this case where $h=h^{\varphi}$, Theorems 2.1 and 2.2 are still true. Nevertheless, the proof of Lemma 2.9 is not correct in the stationary case.

Theorems 3.1 and 3.2 are obviously true for any $d \in \mathbb{N}^{*}$; nevertheless the application to the system (13) is no more possible since the assumptions of these theorems are satisfied by the law of system (13) only if $d \leq 3$.

The results presented in this paper are contained in my Ph.D. Thesis [6] written under the direction of Sylvie Rœlly whom the author thanks warmly for many advises and for her permanent support. The author also thanks the Laboratoire de Statistique et Probabilités of University of Lille 1 for its welcome.

\section{REFERENCES}

[1] S. Albeverio, Yu.G. Kondratiev and M. Röckner, Analysis and geometry on configuration spaces: The Gibbsian case. J. Funct. Anal. 157 (1998) 242-291.

[2] S. Albeverio, M. Röckner and T.S. Zhang, Markov uniqueness for a class of infinite dimensional Dirichlet operators. Stochastic Process. Optimal Control, Stochastics Monogr. 7 (1993) 1-26.

[3] P. Cattiaux, S. Roelly and H. Zessin, Une approche gibbsienne des diffusions browniennes infini-dimensionnelles. Probab. Theory Related Fields 104-2 (1996) 223-248.

[4] P. Dai Pra, S. Rolly and H. Zessin, A Gibbs variational principle in space-time for infinite-dimensional diffusions. Probab. Theory Related Fields 122 (2002) 289-315.

[5] D. Dereudre, Une caractérisation de champs de Gibbs canoniques sur $\mathbb{R}^{d}$ et $\mathcal{C}\left([0,1], \mathbb{R}^{d}\right)$. C. R. Acad. Sci. Paris Sér. I 335 (2002) $177-182$.

[6] D. Dereudre, Diffusions infini-dimensionnelles et champs de Gibbs sur l'espace des trajectoires continues $\mathcal{C}\left([0,1) ; \mathbb{R}^{d}\right)$. Thèse soutenue à l'École Polytechnique (2002).

[7] J.D. Deuschel, Infinite dimensionnal diffusion processes as Gibbs measures on $C[0,1]^{\mathbb{Z}^{d}}$. Probab. Theory Related Fields $\mathbf{7 6}$ (1987) 325-340.

[8] R.L. Dobrushin and J. Fritz, Non-equilibrium dynamics of one-dimensional infinite particle systems with a hard-core interaction. Comm. Math. Phys. 55 (1977) 275-292.

[9] H. Föllmer, Time reversal on Wiener space. Springer-Verlag, Lecture Notes in Math. 1158 (1986) 117-129.

[10] H. Föllmer and A. Wakolbinger, Time reversal of infinite-dimensional diffusions. Stochastic Process. Appl. 22 (1986) 59-77.

[11] M. Fradon, S. Roelly and H. Tanemura, An infinite system of Brownian balls with infinite range interaction. Stochastic Process. Appl. 90-1 (2000) 43-66.

[12] J. Fritz, Gradient dynamics of infinite point systems. Ann. Probab. 15 (1987) 487-514.

[13] J. Fritz and R.L. Dobrushin, Non-equilibrium dynamics of two-dimensional infinite particle systems with a singular interaction. Comm. Math. Phys. 57 (1977) 67-81. 
[14] J. Fritz, S. Roelly and H. Zessin, Stationary states of interacting Brownian motions. Stud. Sci. Math. Hung. 34 (1998) 151-164.

[15] B. Gaveau and P. Trauber, L'intégrale stochastique comme opérateur de divergence dans l'espace fonctionnel. J. Funct. Anal. 46 (1996) 230-238.

[16] H.-O. Georgii, Canonical Gibbs measures. Springer, Lecture Notes in Math. 760 (1979).

[17] H.-O. Georgii, Equilibria for particle motions: Conditionally balanced point random fields, Exchangeability in Probability and Statistics, edited by Koch, Spizzichino. North Holland (1982) 265-280.

[18] E. Glötzl, Gibbsian description of point processes, in Colloquia Mathematica Societatis Janos Bolyai, 24 keszthely. Hungary (1978) 69-84.

[19] E. Glötzl, Lokale Energien und Potentiale für Punktprozesse. Math. Nach. 96 (1980) 195-206.

[20] J. Jacod, Calcul stochastique et problèmes de matingales. Springer, Lecture Notes in Math. 714 (1979).

[21] R. Lang, Unendlich-dimensionale Wienerprozesse mit Wechselwirkung I. Z. Wahrsch. Verw. Gebiete 38 (1977) 55-72.

[22] R. Lang, Unendlich-dimensionale Wienerprozesse mit Wechselwirkung II. Z. Wahrsch. Verw. Gebiete 39 (1977) 277-299.

[23] K. Matthes, J. Kerstan and J. Mecke, Infinitely Divisible Point Process. J. Wiley (1978).

[24] A. Millet, D. Nualart and M. Sanz, Time Reversal for infinite-dimensional diffusions. Probab. Theory Related Fields 82 (1989) 315-347.

[25] R.A. Minlos, S. Rølly and H. Zessin, Gibbs states on space-time. Potential Anal. 13 (2000) 367-408.

[26] X.X. Nguyen and H. Zessin, Integral and differential characterizations of the Gibbs process. Math. Nach. 88 (1979) 105-115.

[27] C. Preston, Random fields. Springer, Lecture Notes in Math. 714 (1976).

[28] N. Privault, A characterization of grand canonical Gibbs measures by duality. Potential Anal. 15 (2001) 23-28.

[29] B. Rauchenschwandtner and A. Wakolbinger, Some aspects of the Papangelou kernel, in Colloquia mathematica societatis Janos Bolyai, 24 keszthely. Hungary (1978) 325-336.

[30] S. Rolly and H. Zessin, Une caractérisation de champs gibbsiens sur un espace de trajectoires. C. R. Acad. Sci. Paris Sér. I 321 (1995) 1377-1382.

[31] D. Ruelle, Statistical Mechanics. Rigorous Results.. Benjamin, New York (1969) .

[32] D. Ruelle, Superstable interactions in classical statistical mechanics. Comm. Math. Phys. 18 (1970) 127-159.

[33] M. Yoshida, Construction of infinite dimensional interacting diffusion processes through Dirichlet forms. Probab. Theory Related Fields 106 (1996) 265-297. 